\title{
RADIOCHEMICAL STUDIES OF NEUTRON DEFICIENT
}

\section{ACTINIDE ISOTOPES}

\author{
ACTINIDE ISOTOPES \\ Apri1 1978
}

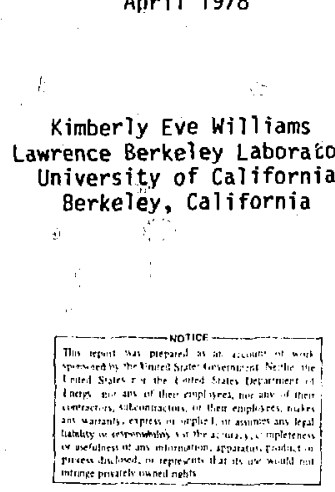


I. INTRODUCTION . . . . . . . . . . . . . . 1

A. Background .................... 1

B. Purpose of this Study ................ i

C. Characteristics of L1ght Berkelfun Isotopes . . . . T:

D. Spontancous Fission Studies............. g

I I. EXPER IMENTAL METHODS ................... 11

A. Target Preparations................. 11

B. Irradiation Techniques .............. 13

1. Recoil Experiments ............. 13

2. Spontaneous Fission Experiments....... 16

III. CHEMISTRY . . . . . . . . . . . . . . . . IB

A. Chemical Properties of Berkelium ......... 18

B. Chemical Properties of Americium and Curium.... I 9

C. Separation Procedures............... 21

1. Berkelium .................. 21

a. TTA Solvent Extraction........... 21

b. HDEHP Solvent Extraction .......... 22

c. HDEHP Column Chromatography ........ 23

2. Americium and Curium .......... 24

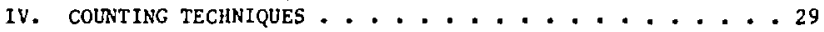

A. Alpha Spectroscopy ................ 29

B. X-Ray Spectroscopy .............. 30

C. Gamma-Ray Spectroscopy .. . . . . . . . . . 31

D. Detection of Spontaneous Flssion Events . . . . 32

v. DATA REDUCTION \& aNALYSIS . . . . . . . . 35

A. Alpha Spectra..................... 35

B. X- and Gamma-Ray Spectra ......... 35

1. Berkellum Samples............ . 35

2. Arser 1cium/Curium Samples .......... 37

C. Spontaneous Flsston Data .......... . 37

D. Cross-Section Estimates............. 39

VI. EXPERIMENTS AND RESLLTS ................. 41

A. Berkelium-242................ 41 4

B. (H.I., pxn) and (H.I., $\alpha \times n)$ Data ........... 5

C. Spontaneous Fission Experiments.......... 47 


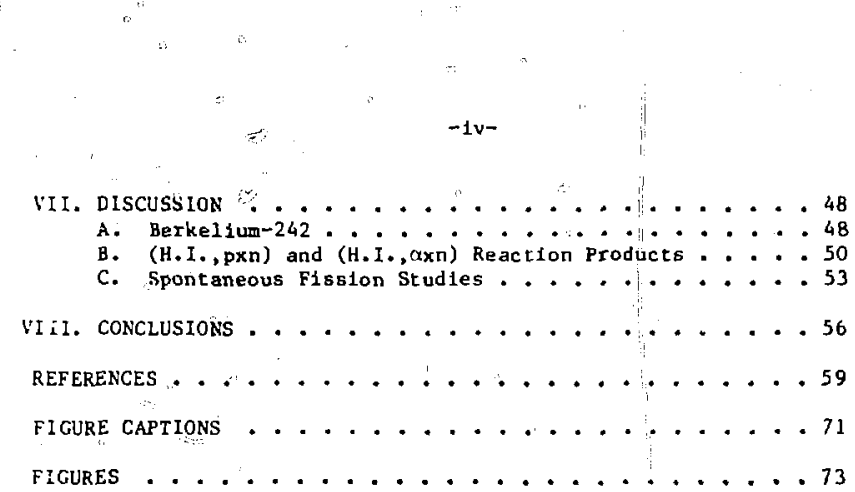




\section{DEDICATION}

This thesis is dedirated to some of the people who have had a profound effect upon my life:

To Shirley for the trips to Echo Lake, her Eriendship and encouragement, and for proving "it could" be done";

To my parents, Lee and Willie, who have continually given me encouragement and support, and who have always taken pride in my accomplishments;

And fina: iy, to Kerl, my flance, for all his confidence in me, his understanding, and his love. 

E. $\quad:-v i-$

There is no heavier burden than a great potential.

- Linus (1970) 
i.

FADIOCHEMICAL STUDIES OF NEUTRON DEFICIENT ACTINIDE ISOTOPES

Kimberly Eve Wil11ams

Nucleat Science Division

Lawrence Berkeley Laboratory

'Iniversity of Callfornta.

Berkeley, CA 94720

\section{ABSTRACT}

The production of neutron deficient actinide isotopes in heavy ion reactions was studied using alpha, gamma, X-ray, and spontaneous fission detection systems. A new 1sotope of berkelfur, $242 \mathrm{Bk}$, was produced with a cross-section of - lupb in reactions of boron on uranium and nitrogen on thorium. It decays by electron capture with a half-life of $7.0 \pm 1.3$ minutes. The alpha-branching ratio for this isotope is less than $1 \%$ and the spontaneous fission ratio is less than $0.03 \%$.

Studies of (Heavy Ion, pxn) and (Heavy Ion, axn) transfer reactions in comparison with (heavy ion, $x n$ ) compound nucleus reactions revealed transfer reaction cross-sections equal to or greater than the compound nucleus yields. The data show that in some cases the yieid of an isotope produced via a $\left(H . I_{., p \times n)}\right.$ or (H.I., axn) reaction may be higher than its production via an $x n$ conpound nucleus reaction. These results have dire consequences for proponents of the " $z_{1}+z_{2}=z_{1+2}$ " philosophy. It is no longer acceptable to assume that (H.I.,pxn) and (H.I.,oxn) product ylelds are of no consequence when studying compound nucleus reactions.

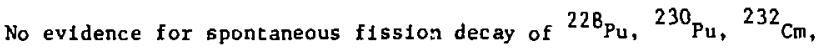
or ${ }^{238} \mathrm{Cf}$ was observed Indicating that strictly empirical extrapolations of spontaneous fission half-11fe data is inadequate for predictions of half-lives for unknown neutron deficient actinide isotopes. 
- ACKNOLILEDGENTS

While many people aided me during my graduate career, sope of the spectal people deserve special thanks:

Ph1l1p Friedman, who got me started in chemistry back in high school and has encouraged me throughout my studies;

flen Seaborg, who as wy research director provided me with unique scientific opportunities and gave me the freedom to choose among them;

The SHEIKS research group for both sclentiric and extracurricular contributions, espectally "LuNCH!!";

Diana Lee for all her computer help and her smile;

Ken Thomas, who went above and beyond the call of duty by aiding me on many owl shifts when he would have prefej red to be home sleeping;

The HILAC staff with whot I worked during my first years at Berkaley - especially Lillian Hill who always loo'ed after me and even let me go through the mail!;

Albert Ghlorso and Matt 1 Nurmia for allowing me to draw on thelr vast experience and for their confidence in me;

The 88-1nch cyclotron staff and operators, especially Roy Benedict, without whom I would have had no beam;

Last, but certainly not least, Harry Harrington and "Cal" Calhoon, the "Bobsey Twins", for keeping me "uncontaminated" (with radiation), for the jokes and : alghs, and for all the times they rescued me from seemingly dire straits!

This work has been supported by the Atomic Energy Comission, the Energy Research and Development Administration, and the Department of Energy. 


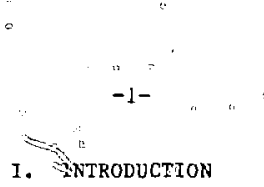

I. INTRODUCTION

\section{A. Background}

When an energetic charged particle approaches a resting tafget nucleus, a wide variety of reactions can take place. The vast majority of the projectiles will lose energy" by interactions with the atomic electrons of the target material. When a particle passes close enough to the target nucleus to be repelled by Coulomb forces (assuming a posftively charged particle) the particle will experience a change in direction and a small loss in kinetic energy (Coulomb Scattering). Projectiles following a grazing trajectory experience the short-range nuclear forces and are either elastically or Inelastically scattered. Those that suffer only a small loss in $k$ inet lc energy and leave the target nucleus in the ground state are elasticaliy scattered while those leaving the target in an exciced state (resulting in a loss of kinetic energy by the projectile) are inelastically scattered. In a few cases the projectile may undergo a muclear reaction with the target nucleus. That is, al1 or part of the projectile may be captured by the nucleus; or the projectile may absorb or strip away part of the target nucleus. These compound nucleus (complete fusion) and transfer (1ncomplete fusion or stripplng) reactions are the interactions of primary concern in this work.

A number of phenomena characterize comnound nucleus formation. 1-3 A primary requirement for the existence of a compound nucleus is that al1 the nucleons from the projectile lose their previous individual and collective characteristics and take on the new characteristics of 


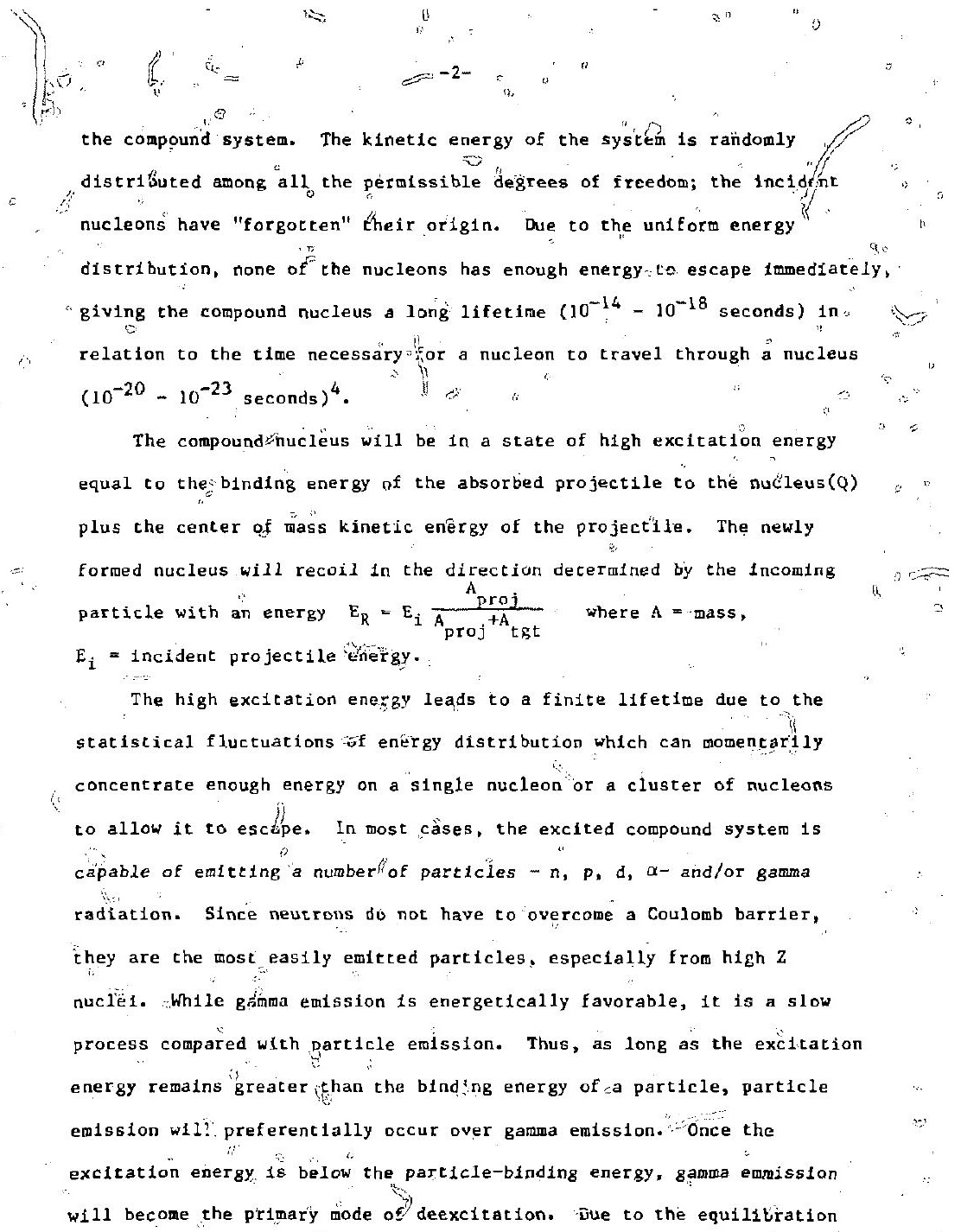


$$
c
$$

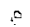
$0=0$

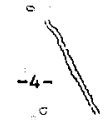

$\operatorname{restriction}$ but at a lower exctitation energy, $\mathrm{E}^{\star}-\frac{\mathrm{J}^{2} \mathrm{~h}^{2}}{2 J}$, whered. Jis the moment of 1nertia. Sinced, is smaller for a $a_{\bar{o}}$ sphere than for

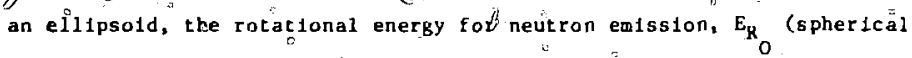
shape), 1s larger" than that "for fission, $E_{\mathrm{R}_{\mathrm{S}}}$ (saddle point shape). The neutron width to fisision width ratio then becomes:

(?)

$\Rightarrow \quad$ a) $\frac{\Gamma_{n}}{\Gamma_{f}}=C \exp \left(\frac{-B_{n}-E_{R_{0}}+B_{f}+E_{R_{S}}}{T}\right)$

Finally, a Totating liquid drop fission barrier can be defined as

$$
\begin{gathered}
B_{f^{\prime}}=B_{f}-\left(E_{R_{0}}-E_{R_{S}}\right) \cdot T_{0}, \\
\quad \frac{\Gamma_{n}}{\Gamma_{f}}=C \exp \left(\frac{B_{f_{R}}-B_{n}}{T}\right) \quad \text { becomes sma } 1
\end{gathered}
$$

when $B_{n}=B_{f_{R}}$ and negligible when $B_{f_{R}}=0$. Therefore, high angular momentum, in addicion to decreasing the total fusion cross-section, $9-11$

- leads to high fission yields of those compound nuclet that are formed.

In the very heavy region $(A \gtrsim 200)$ the fission barriers without

ii rotational considerations are already below $15 \mathrm{MeV}$ and $B_{f}$ eastly decreases to below $B_{n}$. Th1s leads to the expectation that the complete fuston cross-section should be very fiearly the same as the f1ssion cross-section and very few compound nuclei should survive the competitiun with fisston. 12 It must be kept in mind that at each step in the evaporation process, $1 . e$. every time a neutron is emitted, fission is competing. There results, in general, for high $Z$ and $A, a$ decrease $1 n$ yield as more and more neutrons are emitred, 1.e. $o_{\max }(4 n)>\sigma_{\max }(5 n)>$ etc. 'The excitation functions for compound nucleus reactions exh1bit a maximum cross-section with roughly a gaussian shape (F1gure 1). At too low energles, not enough excitacion energy is imparted to emit the desired number of 

neutrons while at higher energies, too much excitation energy is present, resulting in the emission of additional neutrons. The energy of the maximum cross-section is in part a function of the Coulomb barrier and the neutron binding energy:

While the exact mechanisms for transfer reactions are not known, certain properties, characteristic of these reactions, serve to distinguish them from the compound nucleus mechanism. 13-18 The differential cross-section for transfer products peaks at center of mass angles greater than $10^{\circ}$ for bombarding energites near, but above the coulomi barrier. The peaks of the excitation functions generaly occur at higher energies, are more slowly decressing or actually plateau at higher excitation energy, and at times have higher crosssections than those for compound nucleus formation (for the same measured isotope): For the campound nucleus system, the range of the recoiling nuclei increases with increasing bombarding energy, while such a trend is not the case with transfer reactions. For example, Hahn et aI. 17 measured the recoil range of californium isotopes from $1{ }^{2} \mathrm{C}+{ }^{239} \mathrm{Pu}$ and found their ranges to vary from 1.6 times the expected complete fusion value at $67 \mathrm{MeV}$ for this system down to 0.6 of the complete fusion value expected at $97 \mathrm{MeV}$. A characteristic similar to the compound nucleus mechanism is a general trend toward neutron deficient spectes resulting from neutron emission from the excited, newly-formed transfer product. 13 One possible mechanism for these transfer reactions $13-17$ involves a two-step process. In the first step, a cluster or a single nucleon is transferred from the projectile to the target nucleus forming a recoll nuileus with a particular recoll velocity, $\vec{v}_{R_{1}}$, in the direction 
dictated by the incoming projectile $\left(0^{\circ}\right)$. In the secono step, scattering between the recolling nucleus and the remaining light residue gives rise to a velocity, $\vec{v}_{\mathrm{R}_{2}}$, and $a_{\text {scattering angle of }}$ for the recoil nucleus. The final velocit; of the recoiling nucleus, $\vec{v}_{R_{f}}$, is the vector sum of $\overrightarrow{\mathrm{v}}_{\mathrm{k}_{1}}$ and $\overrightarrow{\mathrm{v}}_{\mathrm{K}_{2}}$. Using lihis model Hahn and co-workers 17 were able tó reproduce: the observed experínental trends of recoll range, Ar. angular distribution, average recoll energies, and excitation energies of californium isotopes produced in the ${ }^{12} \mathrm{C}+239 \mathrm{pu}$ reaction. A second possible mechanisim proposed by Galin et al 19-20, requires the transfer to occur at the point of closest approach, $k_{m i n} 1 . e .$, the Incoming and outgoing trajpctorits are expressed solely in terms of Rutherford scattering. Calculations based on this model were also consistent wh the conclusion that the callfornium isotopes measured by than et al. were produced via a transfer of ${ }^{8}$ Be to the plutonium target although a number of detalls in the predictions of the two models are distinctly different.

Information on the mechanisn of production 1s 1mportant in designing experiments to search for new elements and Isotopes since quantitative information on the competing processes in heavy ion reactions can demonstrate which reactions are wost favorable for production of a particular nuclear 1sotope. 21 In addition, the distinct characteristic differences between compound nucleus and transfer reactions have been used to ald In the identification of heavy-ion reaction products. 22 It should be noted, however, that there are some intervals of bombarding energy where recoll energles and angles from compound nuclel and transfer reactions are quite siollar, and great care should thus be taken when 


\section{=

such properties are used to identify new lsocopes. 17

\section{B. Purpose of this Study}

It was decided to investigate the production of neutron defictent actinide isotopes with respect to these reactions. Recencly, exernsive af forts have concentrated on promising mechanisms for the production of superheavy elemeris. 23-31 Very little work, however, has concentrated on investigating the neutron deficient actinides. Berkelium, in particular, has been Ignored. As can be seen from Figure 2 the light berkelium isotopes are "missing". Unt 11 the work presented in this thesls was completed, the lightest isotope of berkelium known was $243 \mathrm{Bk}$, the first berkelium isotope discovered. ${ }^{32}$ (There is some evidence for a spontaneousfy fisstoning 242 Bk 1 somet. ${ }^{33}$ )' Few studies have been made of the berkelium isotopes since the work of Chetham-Strode 34 in 1956 . He investigated the decay properties of berkellum 244-249 and of californium $244-247$.

This work has concentrated on studles of production of the $11 \mathrm{ght}$ berkelium 1sotopes, $242 \mathrm{Bk}$ in particular, and on the possibility of decay by spontaneous fission of light 1sotopes of plutonium, curlum, and californium. It was hoped that through these investigations pertinent Information would be galned concerning competition between fission and neutron emission, the amount of transfer versus compound nucleus reactions, and primary decay modes for neutron deficlent actinide isotopes.

\section{Characteristics of Light Berkelium Isotopes}

The Investigation of berkelium isotopes was undertaken for a number 


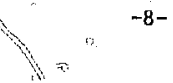

of reasons. As has been pointed out, the light lsotopes of berkelium are missing (Figure 2). Concerngwas expressed as to the reason for this "hole". Was it simply that no one had performed a conscientious searct: for these isotopes; or more interestingly, were they unusualiy shoctlived and/or produced in unexpectedly smail yield? Several years ago some preliminary searches for $11 \mathrm{ght}$ Isotopes of berkelium and curium were giade; but to no aval1. 35 While the parents of the berkelium 1sotopes 239, 241, and 242 are known, these daughter Isotopes have not been observed. ${ }^{36-39} \wedge$ spontancously fissioning 1 somer of ${ }^{242}$ Bk has been reported, 33 but methods of identification based solely on spontaneous fission decay are always subject to question. 40

Ar adtitionally attractive feature of berkelium is its chemistry. Because it exhibits a +4 oxidation state 1 can easily and quickly be removed from the +3 actinldes allowing chemlcal separations to be performed on short-lived 1sotopes. The chemical separation of berkellum is extremely Important both for the identification of the element itself as weII as the Identification of a new 1sotope (via the observation of the daughter of the new species in the parent chemical fraction).

Recent empirical calculations by Kolesnikov and Demin predict an electron capture half-1ife of $13 \mathrm{~min}$ and $27 \mathrm{~m}$ in for berkelium 241 and 242 respectively, and alpha half-lives of 25 and 210 days. 41 These long alpha half-ilves correspond to $\alpha$-branching ratios of 0.0367 and $0.009 \%$ respectively. The emplrically extrapolated alpha energles would be $7.1 \mathrm{MeV}$ and $6.84 \mathrm{MeV}$. Keller and Müzel also predict similar halflives. 42 Since these berkeliums are predicted to decay primarily by electron capture, their Identification had to be based on the characteristic 
$x$-rays of their curium daughters. This necessitated a short, (due to short half-1ives) clean chemical separation of berkelium from isotopes having $X$ or gamma-rays near the $K X$-rays of cur 1 um. The chemical separations used are described in Section III.

There are no reported cross-sections for the production of neutron deficient berkelium isotopes by lons any heavier than alpha particles. The decistor, then, as to what target/projectile combinations to use was based on the cross-section predictions of Alonso 74 described in Section $V-D$. Boron on uranium was chosen as: the most suitable combination due to the large cross-sections predicted (see figure 1), the high Intensity boron beams avallable at the 88 -inch cyclotron, and the ready avallability of various uranium isotopes for targets. Nitrogen on thorium, although not as high in predicted cross-section, was another suitable combination. Alphas and procons were not considered as projectiles because they would not have supplied enough energy to allow the recoils to escape from the target, thus necessitating dissolution of the highly $\alpha$-active, irradiated target.

\section{Spontaneous Fission Studies}

The spontaneous fission studies of plutonium, curium, and californium were undertaken to investigate the possibility of the existence of a spontaneous fission 1 sland in the very neutron deflctent region of these actinides. ${ }^{244} \mathrm{Fm}$, the 11ghtest known fermium 1sotope, decays by spontaneous fission with a half-life of $3.3 \mathrm{msec} .232 \mathrm{Am}, 234 \mathrm{Am}$, and $227 / 228_{\mathrm{Np}}$ are also reported 43 to decay by apontaneaus fission. Thus, there has been sone apeculation $44 a, b, c$ that there may exist a region of spontaneous 
fission "activity in the neutron deficient area. Interest also exists In seeing how the spontaneous fission half-lives of these light actinides might compare to empirical extrapolations from the known isotopes.

The systematics of the spontaneous fission half-lives for even-even isotopes of elenents from plutoni um to nobelium are shown in Figure 3 . Not only does the 152 -neutron subshell produce a well-known effect on the spontaneous fision half-11ves, tut. it also shows up as a chararteristic kink in the alpha energy systematics kith an alpha energy minimum around 152 neutrons (Figure 4). The dashed 1 ines in Figure 3 show purely systenatic predictions obtained by fitting a parabola of the form LOC $T_{1 / 2}=\bar{A}+B\left(N-N_{G}\right)^{2}$ to known points for plutonium, curium, and californius. These predictions do not take into account the fact that the light isotopes, plutonium in particular, are expected to approach spherical shape as the 126 -neutron subshell is approached and, therefore, may not have spontaneous fission half-lives as short as these predictions. It should be kept in mind, however, that the more neutron deficient an isotope is, the more -unstable $1 \mathrm{t}$ is. It is quite possible that the effect of B-instability may completely override any contribution due to the 126-neutron subshell and these 1ight isotopes may, Indeed, have extrenely short half-lives. The spontaneous fission experiments in this work were undertaken in an attempt to provide information on the above question and to investigate, more generally, the properties of the $11 \mathrm{ght}$ actinides. 


\section{EXPERIMENTAL METHODS}

\section{A. Target Preparations}

A number of techniques ${ }^{45}$ were employed to prepare suitable targets for the various bombardments. These included electrodeposition, centrifugation, vacuum evaporation, and electrospraying. The particular technique used was determined by the target materlal itself, the amount of material available, tice desirea thickness and size of the target.

Electrodeposition techniques ${ }^{46-50}$ were used to make thin, uniform targets of uranium and lanthanide elements. Targers thicker than $\sim 500 \mu g / \mathrm{cm}^{2}$, however; were very brittle and easily cracked and Elaked off the target backing. Thick targets were made by plating the target material (lead or bismuth) directly onto the rotating trum used in the cpontaneous fisston experiments.

Targets of various heavy elements such as lead were prepared by centrifugation of a very fine sulfide precipitate in a special centrifuge cone which had the target backing mounted at the hottom of the cone. These cargets were fairly thlck $\left(\sim 1-2 \mathrm{mg} / \mathrm{cm}^{2}\right)$. They were, unfortunately, very fragle and could not generally be used more than a few times. For the spontaneous fission experiments, HgS was painted in a thin filw directly oilu thie drum. Vacuum evaporation $42,52,53$ was a successful technique for nonradloactive targets and for those targets having a large supply of starting material avallable. This technique suffered from large contamination problems which also made recovery of unused starting waterial very difficult.

In an effort to overcome the varlous disadvantages of the techniques 
mentioned above, ancthcr mathod of target preparation was developed and adapted for use with radioact fve materials. The technique is known as electrospraying. ${ }^{54-61}$ A decalled account of the methods used and developed can be found in reference 61, but a simple explanation follows. A schenatic of the apparatus is shown in Figure 5. The target material, in any soluble form - usually the nitrate form, was dissolved An acetone and pipetted into the glass capillary. A voltage of 4500-5000v, $D C$, was applied to the wire extending down the center of the capillary. The acetone solution formed a fine spray which was deposited on the carget backing. The target was at ground potential and several hundred degrees, $C^{\circ}$. Mica screens were used to collimace the spray and a certain amount of focusing of the spray was posstble by applying a voltage to a metal ring set just in front of the target. The result was a uniform layer of target material in oxide form on the target backing. Once the target was finished, it was heated to red hot in an induction coil to insure all the target material was in the oxide form.

Using this procedure it was possible to produce targets ranging from a few $1 \mathrm{~g} / \mathrm{cm}^{2}$ to several $\mathrm{mg} / \mathrm{cm}^{2}$. Heryllium was the typical target backing material although havar, dural, nicke1, and aluminum have also been used. Electrospraying had advantages over a number of other targetmaking techniques in that the oxide targets prepared in this maner were extremely sturdy and uniform up to $5 \mathrm{mg} / \mathrm{cm}^{2}$. Only small amounts of material were needed as the plating efficlency was quite high. The system was easily adapted for use in a glove box and thus radioactivity was not a problem. This method proved to be the most successful and reliable of those used. Consequently almost all targets used in this 
The typical target used in these studies was composed of the oxide form of he target marerial on 0.5 or 1.0 wil Be foll. The targets were $5 / 16$ inches in diameter and approximately $400^{\circ g} / \mathrm{cm}^{2}$ thick. Since a large number of experiments used a ${ }^{235} \mathrm{U}$ target, an analysis of the isotopic composition of the ${ }^{235} \mathrm{U}$ material (obtatned from Oak Ridge National Laboratory) was performed using both a-counting techniques and mass spectrometry. ${ }^{62}$ The target material was determined to be $94.1 \pm 0.12^{235} \mathrm{v}$ and $5.7 \pm 0.12^{238} \mathrm{u}$.

\section{B. Irradiation Techniques}

1. Reco11 Experjments

In an effort to minimize the radiation exposure to the chemist, 1t was declded to catch the recoiling compound nuclet (in experiments where chemistry was to be performed) on a thin catcher foll placed directly behind the target. This meant that only the catcher foll needed to be dissolved and the target could be reused any number of times. Further, the absence of macro amounts of target material greatly simplified the chenlstry. The targets were usually thinner than the recoll range of the compound nuclei so that alwost all the compound nuclei would escape. The catcher foll, on the other hand, was always a minimum of twice the recoil range of the compound nuclei to insure that all the recofling compound nuclel were caught. The ranges were calculated from the tables of Northcliffe and Sch11ling. 63

All irradiations were carrled out in the high level cave area of the 88-inch cyclotron at Laurence Berkeley Laboratory. Figure 6 shows 
a schematic of the experimental set-up. A l/4-1nch diameter, water-cooled collimator was located approximately 4.5 1nches upsteam from the target. Since this collimator was smaller than the target area, all beam measured in the Faraday cup passed through the target. The target was cooled with liquid nitrogen gas flowing between a beryllium window upstream and the target itself downstream. The catcher foll was placed $\sim 0.5 \mathrm{~cm}$ from the target and was cooled with $\sim 150$ torr of hellum. This pressure of helium resulted in less than $0.2 \mathrm{MeV}$ loss of energy for the recolling compound nuclei. 63 That this pressure, indeed, did not alter the collection of recolls was shown by running in a vacuum with the same results as with helium. On occasion, the catcher foll was moved back and a collimator placed in front of 1t. This geometry discriminated against strongly non-forward peaked (1.e. non-compound nucleus) reaction products such as fission früments and transfer products. In addition, while thicker than the compound nuclel recoll range, the catcher foils were still thin enough to allow the more energetic fission fragments and transfer products to pass right through, thereby further discriminating against non-compound nucleus products.

The amount of beam hitting both the collimator and the target - which were electronically isolated from one another - was continuously measured. This enabled the cyclotron operators to optimize the beam by minimizing the collimator reading while maximizing the target reading. To avold inaccurate beam readings, two rare-earth magnets were mounted in front of the target to keep secondary electrons gentisted from the target contained there, while at the same time steering away secondary electrons generated elsewhere. 


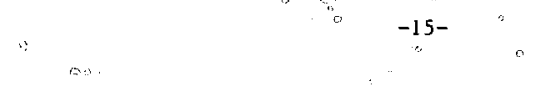

A 3-phase, $60 \mathrm{~Hz}$ beam wobbler was installed about 5 meters upstream from the target (after the last quadrupole vagnet). This device is simply the stator of an induction motor and moves the beam in a small circle at a $60-\mathrm{Hz}$ rate with a loss in beam intensity of $\sim 15 \%$. The use of the wobbler prevented the narrowly-focused beam from depositing a large amount of energy in a small target area and thus helped avold excessive heating of the target in any one spot.

The targets used routinely withstood steady currents of 3 - 5uA of ${ }^{+\hat{3}_{B}}$ for several hours. In a number of instances they withheld beam bursts in excess of $15-204 \mathrm{~A}$, but generally it was considered unsafe to operate at currents higher than 5-8uA. An interlock system was used which automatically cut the Rf power of the cyclotron if the bear current went higher than some preset current or if the target broke (measured as a pressure rise in the beam 11ne). As an additional precaucion to protect the cyclotron fron possible radioactive contanination due to breakage of a radioactive target, a slammer-valve 64 was lesigned and installed $\sim 9.5$ meters upstrean from the target. Even a slight pressure rise In the bean line caused this valve to close within 3 msec, isolating the cyclotron from the target.

The energy of the cyclotron bear was determined by the frequency and the magnetic field of the cyclotron using the following relation:

$$
\omega=(\text { har }) 48.616 \sqrt{1-\frac{1}{\left(1+\frac{E}{m c^{2}}\right)^{2}}}
$$

where $\omega=$ cyclotron frequency in megacycles/sec, $E=$ bean energy in Mey, har $*$ harmonic, $\mathrm{sc}^{2}=\mathrm{A}$ of beam $x(931.478)-q \times(0.511)$, and 
q = charge state of beam. Th1s method was chécked independent ly with a magnetic analyzer and found to be quite accurate. The final energy of the beam in the target was calculated from the electrontc stopping powe $r$ tables of Northcliffe and Schilling, 63 eaking ints account the beam window-havar, aluminum or beryllium; the nitrogen cooling gas; the target backing (beryllium) and the target materdal itself. The bombarding energy was" alcered by using various materlais and thicknesses for the beam window. In none of the cases considered was the beam energy : degraded more than $13 \mathrm{MeV}$. The energy loss in the target was typically $0.5 \mathrm{MeV}$.

\section{Spontaneous Fission Experiments}

The technique for detecting spontaneous fission events from the decay of compound nuclel entalled either 1) the collection of recoll atoms on the surface of a rotating drum in cases where a thin target was used; or 2) plating or coating the drum surface with carget material which then acted as a thick target and a collector for the recolls. Surrounding and facing the drum surface were strips of muscivite mica which served to record the fission fragment tracks (Figure 7). The retoil atoms were caught on the drum and carrled unt1l they decayed, Ieaving tracks in the mica. These tracks were easily visible at loox magnification after etching in hot hydrofluoric acid.

The targets were of two forms: thin (less than $1 \mathrm{ig} / \mathrm{cm}^{2}$ ) targets on beryllium, aluminum, or havar backings or the drum itself was made to be the target by coating it with a thick (several $\mathrm{mg} / \mathrm{cm}^{2}$ ) layer of target material. The targets were prepared using the methods previously 



\section{A. Chemical Properties of Berkel'ium}

As do all thectransurantum elements, berkelium exhiblas the $t_{3}$ oxidation state in solution; Bk(111) is carried by $\mathrm{LaF}_{3}$ or $\mathrm{La}_{\mathrm{O}}(\mathrm{OH})_{3}$ while berkelium nitrate, sulphate, chloride, sulfide and perchlorate are solutle In water. Bk(III) can be readily complexed with thenoyltrifluoracetone (TTA) and extracted into benzene or xylene, and at $\mathrm{pH} 4$ jeparation from curlum and anerictum can be effected. ${ }^{65}$ Moderately strong complexes can also be formed with citrate, lactate, tartrate, $\alpha$-hydroxyisobutyrate; ethylene diamine tetraacetic acid (EDTA), acetate, and thlocyanate. Bk(III) follows closely the fon exchange behavior of its lanthanide homologue - terbium. Figure 8 shows the rates of elution of californium, berkelium, curium and americium compared with their lanthanide homologues on Dowex-50 resin (cation exchange) and citrate cluant at $87^{\circ} \mathrm{C} .66$ A distinct break occurs between gadolinium and terbium and also between curium and berkeliun as a consequence of the abrupt change in ionic radius which occurs upon completion of the $4 \mathrm{f}^{7}$ shell in gadolinium and the $5 \dot{f}^{7}$ she 31 in curtum. This discontinuity in ionic radius is manifested as a significant change in complex ion firmacion resulting ip a gap in the elution curves. Berkelium elutes ahead of the lanthanide elements on a saturated HCL-Dowex-50 column 67 indicating that berkelium forms a stronger" chloride complex than do the lanthanides. Berkelium is also held, albeit weakly, on a Dowex-l column (anton resin) in saturated $\mathrm{HCl}^{68}$ demonstrating substantial formation of anionic chloríde eumplexes. 
Berkelium's lanthanide homologue, terbium, exhiblts not only a $t_{3}$ oxidation state, but (in the solid state) a ${ }_{4}$ state as wel: $\left(4 f^{7}\right.$ ). Berkelium achleves the particularly stable half-filled $5 f^{7}$ structure by also assuming a ${ }_{4}$ oxidation state. Further, in general, higher oxidation states are more easily realized in actinide elements than in lanthanides; thus the $+_{4}$ stace of berkelium is stable in aqueous solution as well as in the solid phase. This is a particularly convenient property of berkelium because it allows Bk(IV) to be raptdly and quantitaively extracted into such reagents as bis(2-eihylhexyl orthophosphoric acid (HDEHP)/heptane frow nitric acid leaving behtind the ${ }^{+}{ }_{3}$ lanthanides and actinides, 69,70 Bk(IV) can also be extracted quantitatively into TTA/xylene ${ }^{71}$ with excellent separation from alkalles, alkaline earths, trivalent lanthanides, $\mathrm{Ru}, \mathrm{Zr}, \mathrm{Nb}, \mathrm{U}, \mathrm{Np}, \mathrm{Pu}, \mathrm{Am}$, $\mathrm{Cm}, \mathrm{Fe}, \mathrm{N} 1, \mathrm{Al}$, and $\mathrm{Ag}$.

\section{B. Chemical Properties of Americium and Curium}

Americium is the actinide homologue of europium and most comonly exhibits the propertes typical of a trivalent actinide or lanthanide. other valence states, namely 0 , IV, $V$, and VI have been found, but only An (III), (V), and (VI) are stable in aquepus solution. The higher valence states may, at times, be used to effect chemical separations of americlum from curium.

Curium is the actinide homologue of gadolinfum and exhibits a single valence state of ${ }^{+} 3$ in solution. Thus curium follows the expected behavior of trivalent lanthanides and actinides toward group reagents and is very similar to trivalent americium. Curium (III) and americium (III) 
quancitatively precipitate as the fluoride, hydroxide, and oxalate. The ${ }^{+}{ }_{3}$ actinfdes tightly bind to cation exchange resin and moderately strong complexes are required to remove them. The ${ }^{+}$actinides elute ahead of the lanthanides from Dowex-50 restin in saturated HC1 67,72 making possible an effective group separation of the actinides and 1anthanides. Cm(III) may be separated from Am (III) on a Dowex-50 column at $87^{\circ}$ with $\alpha$-hydroxylsobutyrate. 73 There is little or no tendency for Am (III) or Cm (III) to absorb onto anion resin from $0.1 \mathrm{M}$ to concentrated $\mathrm{H}_{2} \mathrm{SO}_{4}$, $\mathrm{HCl}$ or $\mathrm{HNO}_{3} \cdot 68,74,75$

Both americium (III) and curium (III) can be extracted into TTA/benzene at pH $>3 .^{65}$ Goffart and Duyckaerts ${ }^{76}$ have studled the extraction of curium (III) and amertcium (III) from nitric acid solutions by tri-n-octylphosphine oxide, TOPO, In benzene and also by tri-n-butylphosphine oxide, TBPO. Curium and americtum may be separated by a solvent extraction procedure using tributal phosphate, TBP, developed by Peppard and Gray. 77 Curium (III) is extracted to a greater extent than americlum (III) and is concentrated in the organic (TBP) phase by use of countercurrent methods, while americium (III) migrates to the aqueous (10-16M HNO 3 ) phase.

For a more detailed accounting of the chemistry of the actinide elements, the reader 15 referred to references 78-84 which are on $1 y$ a small sample of the multitude of publications concerned with actinide chemistry . 


\section{Separation Procedures}

\section{Berke11um}

A varlety of rhemical separations of berkellum from the catcher folls was explored before the final chemistry was chosen. Speed was one of the primary considerations. Searching for half-11ves less than 20 minutes (ultimately $\approx 7$ min) ruled out the use of an a-hydoxyisobutyrate/Dowex-50 column. In fact, the time element ruled out most of the more classic separation schemes almed at isolating berkelfum (III). Attention was therefore focused on the separation of berkelium (IV). Berkelium (IV) can easily be extracted away from ${ }^{+}{ }_{3}$ actinides, ${ }^{+}$ lanthanides, Pu, $U, T h, A l$ and a score of other elements in a very short time (< $5 \mathrm{~min}$ ) compared to the more involved procedures necessary for berkelium (III) separation. The following procedures were all employed in the course of this work. The HDEHP solvent extracton procedure, however, was determined to be the most efficlent and practical separation considering the various experimental constraints. It is important to note, however, that all three procedures gave consistent experimental results when corrected for chemical yleid and the time from the end of bombardment. Additional procedures for the separation of berkelium (IV) may be found in references $85-91$.

\section{a. TTA Solvent Extractions}

The procedure of F.L. Moore 71 was adapted for speed and small volume work, but essentlally remained unchanged (Figure 9). The Al catcher* resulting from products due to boron and nitrogen on $\mathrm{N1}, \mathrm{Cu}, \mathrm{Cr}$, etc. 
was dissolved $1 \mathrm{n}$ a $\mathrm{mln}$ imum amount of $7 \mathrm{M} \mathrm{HCl}$, dried, converted to nitrate form with 1 drop of $8 \mathrm{NHO}_{3}$ and deled again. $450 \mathrm{~h}$ of

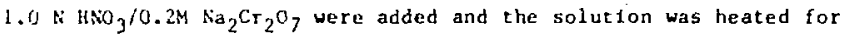
1 minute. 502 of $5 \mathrm{~N} \mathrm{H}_{2} \mathrm{SO}_{4}$ were added and the solution cooled quickly in an ice bath. 500ג of $0.5 \mathrm{M}$ TTA/xylene were added to the aqueous solution and the two phases wert mixed using a Vortex mixer for $\sim$ minute. The aqueous phase was removed and the organic phase was washed with 500 a of $1.0 \mathrm{~N} \mathrm{H}_{2} \mathrm{SO}_{4} / 0.2 \mathrm{M} \mathrm{Na}_{2} \mathrm{Cr}_{2} \mathrm{O}_{7}$. The aqueous wash was removed and the organic phase was contacted with $250 \mathrm{~A}$ of $10 \mathrm{NH}_{3}$. The berkellum was found in this aqueous back-extraction phase. "It was then plated on a platinum disc for $a, Y$, and/or $X-r a y$ counting. To fucther speed up this separation, the wash of the organic phase and the back extraction were climinated and the volume of the organic phase was cut to $250 x$. The cherical yleld after these modifications was $\sim 50 \%$. Th1s particular separation is valuable because, unlike the separations based on HDEHP, this procedure effects a separation of berkelium (IV) from Ce (IV), $\mathrm{Pu}$ (IV), $\mathrm{Np}$ (IV) and $U$ from aqueous solutions of $\mathrm{HNO}_{3}$ and to a lesser degree from $\mathrm{H}_{2} \mathrm{SO}_{4}$, or $\mathrm{HCl}$. Unfortunately, however, thls chem1stry extracts ${ }^{233} \mathrm{~Pa}$ which is produced in abundance from a ${ }^{235} \mathrm{U}$ target and even worse, has a $111 \mathrm{KeV}$ gamma ray which interferes with the observance of the $C_{m} K_{a_{1}}$, X-ray ( $\left.109.29 \mathrm{KeV}\right)$. Thus this chemistry was useful only for alpha analysis of the berkeltum fraction.

\section{b. HDEHP Solvent Extraction}

A number of authors have reported procedures for the extraction of Bk(IV) by b1s(2-ethyllexyi) orthophosphortc actd -- $\left(\mathrm{C}_{8} \mathrm{H}_{17} \mathrm{O}_{2} \mathrm{PHOH}\right.$, 
HDEHP. $70,80,81,92-95$ Using this extractant under the appropriate conditions, tetra- and hexavalent lanthanide and actinide lons may be removed from an aqueous nitric acid phase Into the HDEHP while the trivalent lons present remain preferentially in the aqueous phase. Thus this technique is not sultable for separating Bk (IV) from Ce (IV). or macro amounts of $U$ (VI) or Pu (IV). On the tracer scale, however, urantum and plutonium were not significant problems.

The procedure of Peppard, Moline, and Mason 92 was found to be quite efficient (60\%) even after it was modifled for time (all mixing or equilibration steps were cut to $\sim 1 \mathrm{~m} I n$ ) and volume (volumes used were on the order of $250 \mathrm{~A}$ ). It had one major drawback in that the berkelium was oxidized by $\mathrm{KHrO}_{3}$ in $1 \mathrm{OM} \mathrm{HNO}_{3} \cdot \mathrm{KBrO}_{3}$ is unstable at such a high acid concentration requiring the $\mathrm{KBrO}_{3} / \mathrm{HNO}_{3}$ solutions to cont inually be freshly prepared. Further, the release of browine gas from the solution required special, careful handling in fume hoods and created a generally "nasty" environgent.

This problem was overcome in the separation procedure of Knauer and weaver. 70 In this cage, berkelium was oxidized with chromic acid (Instead of $\mathrm{KBrO}_{3}$ ) in $4 \mathrm{H} \mathrm{HNO}_{3}$. It was then extracted into $0.15 \mathrm{M}$ HDEHP/n-dodecane. The exact procedure adopted for this work, shown in Figure 10, 15 as follows:

1) The aluminum catcher foll was dissolved rapidly in $\sim 1-2$ drops of $2 \mathrm{~N} \mathrm{HNO}_{3}$ in the presence of mercuric ion.

2) 5002 of $0.1 \mathrm{M} \mathrm{CrO} / 4 \mathrm{~N} \mathrm{HNO}_{3}$ were added followed by 5002 of $0.15 \mathrm{M}$ HDEHP in dodecane. The dodecane had prevlously been scrubbed with 
concentrated $\mathrm{H}_{2} \mathrm{SO}_{4}$ to destroy possible contaminants.

3) The phases were rixed for approximately 4 minutes - the tiwe $1 t$ took fer transportation of the solution from the cyclotron to the bullding where the chemlstry was completed and the counting performed.

4) Upon arrival at the chemistry building, the phases were

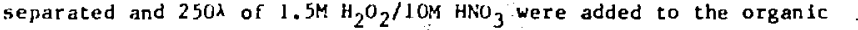
pliase.

5) After mixing for 1 minute, the aqueous phase was cemoved and either taken to dryness on a platlnum dlsc for later alpha analysis or absorbed onto $1 / 4-1$ incly diameter whatman 41 filter paper discs for gamma analysis.:

This procedure proved to be the fastest and most efficient chemistry and was therefore used in the majorlty of experiments. The average time from the end of bombardment to the detector was 12 minutes with a chemical efficiency of $50 \%$.

\section{c. HDEHP Column Chromatography}

Before the final chemistry was chosen, a method for the extraction of berkelium (IV) by liquid-liquid chromatography was devised. It was felt that this form of extraction might be faster and/or more efficient than direct solvent extraction. Both beliefs proved true under appropriate conditions.

The columns (2.5 mm 1.d.) consisted of HDEHP adsorbed on 60-70 mesh tefion powder (obtained from Ken Hulet at Lawrence Livermore Laboratory) as a solld support. 94 The HDEHP-teflon waterial was prepared by slurrying 0.75 grams of teflon with $100 \lambda$ of HDEHP and 10-15 wi of acetone 
with the excess solvent being evaporated at room temperature. The columns were dry-packed to a height of $\sim 5 \mathrm{~cm}$ by gently packing the HDEHP/teflon material into the colman with a glass rod. Prior to packing, the columns were made hydrophoblc by exposure to dichlorodimethyl silane vapors for at least 24 hours.

After packing, a minimum of 10 column volumes of $0,1 \mathrm{M} \mathrm{CrO}_{3} / 4 \mathrm{M} \mathrm{HNO}_{3}$ was run through the column to prepare it for the berkelium extraction. The dissolved catcher foll was loaded in a minimum volume of $0.1 \mathrm{M} \mathrm{CrO}_{3} / 4 \mathrm{M} \mathrm{HNO}_{3}$. Pressure was applied to obtain a flow rate equal

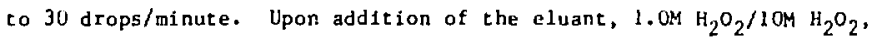
the column turned from orange to green and a significant number of gas pockets were formed in the column bed. A minimum of 5 colum volumes was necessary to remove the berkelium. In spite of the rapid flow rate and disrupted column bed, the chemical yleld was typically $70 \%$. The time for this chemistry was actually shorter than the solvent extraction procedure when both were performed side by side in the laboratory. However, stnce the solvent extraction method could be carried out during transportation to the cheristry buflding, the total time from the end of bombardment to the detector for the solvent extraction method was shorter. In additon, the columns could not be reused and had to be cleaned and repacked with fresh materlal. Thus this procedure was extremely inconvenient for bombardments in which a number of catcher folls were processed in a short perlod of time. However, it was the best procedure used both in chemical yle1d and time for chemistry. Only the constraints due to having to transport the catcher folls to another building resulted in the solvent extraction method being 
favored.

A note about the purffication of the HDEHP is appropriate at this point. Typically, HDEH is commercially provided as a mixture of the di-ester (used in this work) along with up to several percent of mono-2-ethylhexyl orthophosphoric acid, polyphosphates, pyrophosphates, 2-ethylhexanol, other alcohols, and frequently various metal ions such as iron (1II). The monophosphates and the pyrophosphates are the most active impuritles. They require careful removal, as even a trace of these Impuritles greatly increases the distribution coefficients of many elements.

Two sources of HDEHP were used in this wark:

1) Several milliliters of the di-ester, purffied using the techniques of Stewart and Crandal1 96 and Peppard et al., 97 were obtained from Ron Lougheed at Lawrence Livermore laboratory. The original HDEHP was from Eastman Kodak co.

2) $13 \mathrm{DEHP}$, $96 \%$ di-ester, was also obtained from 1CN-KdK Laboratorles, Plainview, NY. Purification of separate aliquots was carried out using the above techriques 96,97 on one aliquot and the alkall washing technique of wureev el al. 93 on another. Since the chemical separation results from all three "batches" were consistent, the procedure of Gureev et al. was preferred since tt was simplest and required the least volume of reagents. For even greater purification, the procedure of MeDowell et al. is recommended. 98

\section{Americium and Curium}

The flow chart in Figure 10 demonstrates the procedure followed 
for the separation of curlum/americium fractions from the catcher folls and from the parent berkelium fraction. This chemistry is a classical transplutonium chemistry based on procedures given in references 81 and 99. The sample was dissolved in $9 \mathrm{~N} \mathrm{HCl}$ and $n$ few drops of water added. After the addition of $-250 \mathrm{\mu g}$ of lanthanum carrier, loud of concentrated hydrofluoric acid were added. After stirring, the solution was allowed to stand for several winutes before centlfuging. The supernatant was removed and the precipltate was washed several times with $2 \mathrm{~N} \mathrm{HNO}_{3} / 2 \mathrm{~N} \mathrm{HF}$ prior to beling dissolved in 2 drops of saturated borlc acld and $150 \lambda$ of concentrated $\mathrm{HNO}_{3}$. This step effectively separated the ${ }^{+}{ }_{3}$ actinides and lanthanides from many contaminating ions of other valences. Thorium, uranium, and plutontum were also precipitated and were separated later on an anion exchange column.

The lanthanides and actinides were removed from the boric acid solution by precipitating chem as hydroxides. To the above solution of boric actd/HNO ${ }_{3}$ lanthanldes/actinides wete added $\sim 12$ drops of concentrated $\mathrm{NH}_{4} \mathrm{OH}$. After centrifuging and removing the supernatant, the precipitate was washed $3-4$ times with water before redissolving it In a minimum volume of $8 \mathrm{NHO}_{3}$. Thorfum, urarifum, and plutonfum were separated by loading the solution on a $2.5 \mathrm{~cm} \times 2.5 \mathrm{mp} 1$.d. Dowex $1 \times 8$ anton exchange column. Dry $\mathrm{N}_{2}$ was used to force $8 \mathrm{~N} \mathrm{HNO}_{3}$ through the column at a rate of $4 \mathrm{drops} / \mathrm{min}$. The lanthanides and actinides were collected in drops 4-20 while plutoniulm, thorium, and uranium rewalned on the column. This solution was taken to dryness, time permitting, or else another hydroxide precipitution was performed as the volume reduction step. To the dried lalAc fraction (or to the 
hydroxide precipitate) was added $\sim$ d drop of saturated HCl followed by $\sim 1 \mathrm{mg}$ of $\mathrm{Ba}\left(\mathrm{NO}_{3}\right)_{2}$. (Radium precipitated with the bariun chloride.) The supernatant was removed and loaded onto a saturated HCl MP-50 column, $5 \mathrm{~cm} \times 2.5 \mathrm{mg} 1 . \mathrm{d}$. , having a flow rate of less than $3 \mathrm{drops} / \mathrm{min}$. The actinide elements eluted ahead of the lanthanides and werc collected in drops 5-35. For a final purification, this solution was taken to dryness, redissolved in $1.5 M \mathrm{HCl}$ and loaded onto another Dowex $1 \times 8$ column, $2.5 \mathrm{~cm} \times 2.5 \mathrm{~mm} 1 . \mathrm{d}$, with a flow rate of $\sim 6 \mathrm{drops} / \mathrm{min}$. Th1s step removed lead and blsmuth daughter products. Drops 4-20 were collected and taken to dryness on a l-inch platinum disc for alpha and/or gamma counting. If this last fraction was contaminated with salts, it was taken to dryness, redissolved in acetate buffer $(\mathrm{pH} \approx 4.5)$ and contacted with an equal volume of $0.2 \mathrm{MTA}$ /benzene several times. 100 The TTA fractions were combined and taken to dryness on platinum. After Flaming the platinum disc, the result was a wefght less sample fdeal for $\alpha$-counting. The chemical yield was typically $75 \%$ but was always measured directly with the use of ${ }^{241} \mathrm{Am}$ and ${ }^{244} \mathrm{Cm}$ tracers. The time for the entire procedure was approximately 100 minutes. 
IV. COUNT ING TECHNIQUES

\section{A. Alpha Spectroscopy}

Alpha spectroscopy 101 of the samples was carrled out using four gold-plated surface barrier detectors obtained from 0rtec, Inc. The detectors hat an average active area of $300 \mathrm{~mm}^{2}$, an energy resolution (FWHM) of $20 \mathrm{keV}$ measured from the $5.485 \mathrm{MeV} \alpha$ peak of ${ }^{241} \mathrm{Am}$, a no $1 \mathrm{se}$ width no greater thin $15 \mathrm{keV}$ (FWHM), a sensitive thickness of -100 microns, and operated at a voltage of 50-100 volts.

The voltage for each detector was supplied through a pre*amplifier which also fed the output signal to a Tranlamp-linear amplifier for alpha detection and to another linear amplifier for spontaneous fission detection (an option not used in these studies). All four signals fiom the linear amplifiers went into a router/mixer. From here, one signal at a time went to a blased amplifier which served to set the window size for alpha detection and the upper and lower window limits (1n MeV). The signal was then fed Into a pulse-height analyzer system consisting of a Texas Instruments $960 \mathrm{~d}$ minicomputer, a Northern Sclentific NS-621 analog-to-d1gital converter, 4096 memory storage locations ( 1024 channels) detector) and an Ampex magnetic tape deck. The data from each decector were stored in 1024 channels, and provisions existed for separate tag-words for each detector. Since all four detectors operated through the same blased applifier and the same clock, they all were set for identfcal gindows and counting intervals. The minicomputer offered a variety of options inciuding integrating counts in varlable channel intervals; recording the tag word, atart time, stop time, live time, and counting 
Interval on magnetic tape; finding peak centrolds; and printing-out the above information on a teletype.

The detectors were callbrated using thin $241 \mathrm{Am}$ and $233_{\mathrm{U}}$ sources in conjunction with a Porpul II $B$ pulser. Least squares analysis of $\sim 10$ polnts between 4.0 and 9.0 MeV gave callbrations with fit parameters $\approx 0 . \overline{99}$ ( 1.000 being a "perfect" $21 t)$. The callbrations were shown to be constant over perlods of several weeks and varled no more than a few channels over longer perlods. The detectors used were brand new at the start of these experiments and had essentlally zero background over counting intervals of up to ten days.

\section{B. X-Ray Spectroscojy}

An intrin-ic germanfum solid-state detector ( $2 \mathrm{cr}$ wide $\mathrm{x} 3 \mathrm{~mm}$ thick) with $\sim 10 \mathrm{mil}$ beryllium window was used in analysis of $\mathrm{X}-\mathrm{ray}$ peaks. The detector was operated in conjunction with a 1024 channel (storage location) Northern Scientific NS-610 multi-channel analyzer, a Northern Scientific N5-400 data processor, and a NS-406M magnetic tape control. The system was callbrated using several isotoplc standards: $241 \mathrm{Am}(59.57 \mathrm{keV}),{ }^{207} \mathrm{B1}(72.79,74,96,84.9,87.4 \mathrm{keV})$, and ${ }^{57} \mathrm{Co}(122.1 \mathrm{keV})$. The energy resolution was $60 \mathrm{c} \mathrm{eV}$.

The absolute efficlency was not deterwined as this method of detection was used only to confirm the Identification of the curium x-rays and to Insure tivat chere were no other conflicting gamma rays present in che spectra. The samples were, however, counted at constant geometry - approximately $0.5 \mathrm{~cm}$ from the berylluw widoe - alloutng half-life measurements, but not absolute cross-section measurements to be made. 


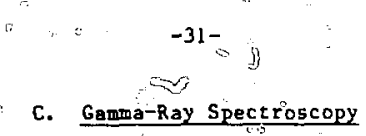

A $60-\mathrm{cm}^{3}$ volume ORTEC coaxial-germanilum lithlum drifted, diode detector was used in the gama and the majority of the $x$-ray analyses. The detectoriwas shielded by $5 \mathrm{~cm}$ of 1 ead, $0.3 \mathrm{~cm}$ of steel and $0.3 \mathrm{~cm}$ of aluminum to reduce the contribution of background radiation and fluorescence inducedin the lead. The detector and pre-amplifier were coupled to a linear amplifier then to a pulse-helghe analysis systew consisting of a Northern Scientific NS-623 analog-to-digttal converter, a Texas Instrument 960A minicomputer, 4096 channels for memory"storage, and an Ampex magnetic tape unit. This system had simiglar capabilities to the alpha counting system, i.e. storage of tag-words, counting intervals, start, stop, and live time on magnetic tape, peak integrations, finding. peak centroids, etc.

Energy calibrations and detector efficlencles were measured using a National Bureau of Standards Mixed Radionuclide Gamma-Ray Edission-Rate Point-Source Standard (SRM 4216-B) which ccntained the isotopes listed In Table 1. least square fits of the channel versus energy data from this calibration source led to fit parameters, as in the alpha system, of $0 . \overline{99}$ ( 1.000 beling a perfect $f i t$ ). The electronics were extremely stable with a calibration shift of no more than $\sim 1$ channel in several months. The resolution of the curlum $\mathrm{K}_{\alpha_{1}} \mathrm{x}$-ray peak $(109.27 \mathrm{keV})^{102}$ was $1.5 \mathrm{keV}$ FWH. The 8 amples were mounted on $8.8 \mathrm{~cm} \times 6.2$ counting cards and covered with a layer of saran wrap to prevent possible contamination of the detectors. All samples were counted $0.5 \mathrm{~cm}$ from the detector face. In this position the efflciency for 110-keV was 15\%. The samples were only ulightly active so the dead time was essentially 
0z. The efficiency as function of energy was previous 1 ' determined for each thelf location ${ }^{103}$ using the procedure of reference 104. A sample spectrum is given in Figure 15 .

Table 1.

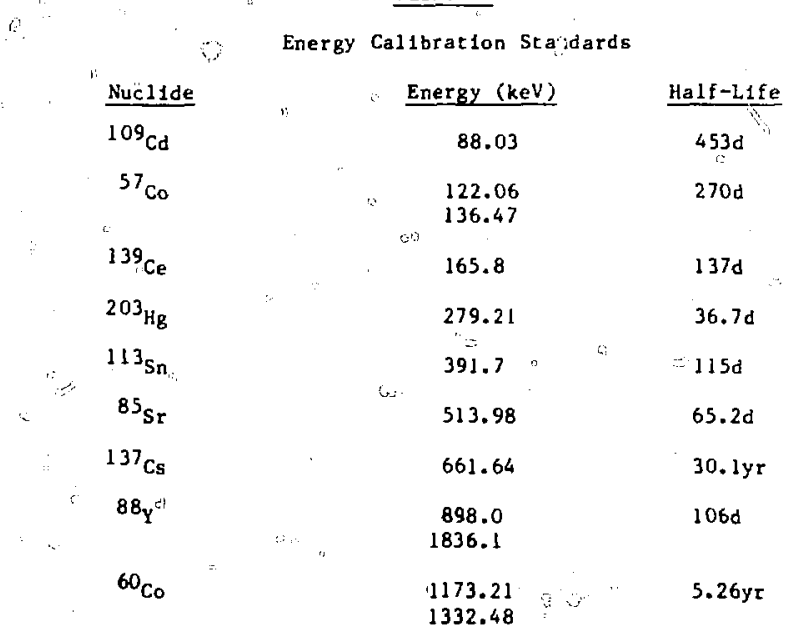

background counts were taken to determine which peaks would be seen regardiess of the sanple being counted. $40_{K}$ along with the natural products of the uranim and thorfum decay chains were the only major background activities noted in seyeral days of counting.

D. Detection of Spontaneous Fission Events

Heavily lonizing, charged particles that pass through wost dielectric (insulating) collds leave narrow tracks of intense demege. 105-109 since this dange 1 s on the atonic scale, it wat be anplified before it can be 
observed. Often this is /iccomplished by cliemical etching of the sol1d, 110 a relatively simple technique. The cheaical etchant preferentially ittacks the damaged area while less rapidly attacking the undamaged aterial, resulting in an enlarging of the damaged area. It is then ossible to observe these holes with an optical microscope (Figure 12).

Three basic types of solids ate readily available for use as particle detectors: minerals, glasses, and organic polymers. For this work, flexible detectors were necessary to facilitate mounting around the drum and later mounting on glass sildes for scanning. Thin mica shrecs and the various organic polymers were especially suiced to this purpise. In some preliminary: experiments both lexan polycarbonate obtained from $P$ Buford Price (LBL) and oica detectors were used in an effort to compace them both for sensitivity, ease of handling and $\varepsilon$ canning. Mica was chosen because of its insensitivity to low $Z$ particles, in contrast to lexan which was sensitive even to alpha particles at $0.3 \mathrm{MeV}$ (reference 111, p. 18, Fig. 1-8).

The mica detectors were split and cut from 4-inch $\times$ 6-1nch muscovite sheets, ASTM-R. 112 Muscovite, $\mathrm{KAl}_{3} \mathrm{SI}_{3} \mathrm{O}_{10}(\mathrm{OH})_{2}$, is the best of all micas In dfelectric strengh, transparency, and perfection of cleavage. After cutting, the micas were annealed at $500 \pm 25^{\circ} \mathrm{C}$ for 4 weeks. 111,113 In addition to annealing, it was also necessary to pre-etcin the miras for 4 hours in concentrated hydrofluoric acid at $60^{\circ} \mathrm{C}$ followed by $1 / 2$ hr rinse in distilled water and a rinse with absolute ethyl alcohol. This preparation piocedure eliminated any background fisston tracks existing in the micas prior to Irradiation. After a bombardment, the micas were masked and individually exposed to a nontransferable ${ }^{252} \mathrm{Cf}$ source to provide 
a check on mica sensitivity and scanning efficiency. They were then etched for 1 hour in concentrated $H F$ at $60^{\circ} \mathrm{C}$, rinsed in water and dried with Et.OH. For scanning purposes, the micas were mounted on $2 \times 3$ inch glass slides and examined at loOX magnificacion (Figure 12). The postions of the flssion tracks were permanent $1 y$ recorded by an X-Y plotter 1nterfaced to the microscope slide. Scanning efflciency was $100 \%$

As a check against tracks originating from fissionable material in the mica (due to the high neutron flux during the irradiation), the back as well as the front slde of each mica was scanned. Corrections were made, when necessary, for any background tracks detected. bith the particular batch of micas used, the average number of background tracks/mica was $\sim 1$. To be sure that deeply buried, unanntaled background tracks were not exposed on the final etch (to look like experimental tracks), 10 micas were annealed, pre-etched, scanned front and back, etched for an additional hour and scanned again. Not a single "experimental" fłssion track was found.

In determining the total detection efficlency of the system, account was taken of the eqissivity of the drum (probability of the escape of one fission fragment from the decay of an atom $-88 \%$ ), loss in the stopper foll $(\sim 4 \%)$, loss due to the $2 \mathrm{~cm}^{2}$ collimator $(\sim 4 \%)$ and decay In the window for very short-lived actfulties. These figures were computed from particle range energy relations at specific trajectorles. 19 As a further check of the system, ${ }^{244} \mathrm{Fm}$ was produced in the reaction ${ }^{233} \mathrm{U}\left(16_{0}, 5 n\right)^{244} \mathrm{Fm}$ with a cross-section in excellent agreement with that previously reported by Nurmia et al, 114 


$$
\text { v. DATA REDUCTION AND ANALYSIS }
$$

\section{A. Alpha Spectra}

Since the samples counted were thin sources, the a-peaks seen were well-separated and we11-defined as shown in Figure 13. The counts in each peak (idelitified by chemlcal fraction, energy, and half-life, if possible) were integrated, an $A_{0}$ determined, and finally a cross-section for production was calculated. This calculation included corrections for chemical yield, length of bombardment, detector efficlency, branching ratio of the particular alpha, etc. Chemical ylelds were determined by adding known count rates of ${ }^{244} \mathrm{Cm}$ and ${ }^{241} \mathrm{rim}$ to the samples before chemistry was begun.

\section{B. $X-$ and Gamma-Ray Spectra}

\section{Berkelium Samples}

Identification of the berkelium isotopes was based on the observance of the $\mathrm{k}_{a_{1}}(109.27 \mathrm{keV})$ and $\mathrm{k}_{a_{2}}(104.59 \mathrm{keV})^{102} \mathrm{x}$-rays of curfum and the hali-lives obtalned from their decay. In cases where the yield of berkelfum isotopes was especially good, the $k_{B}(i 23.41 \mathrm{keV})$ and $K_{B_{2}}(122.29 \mathrm{keV})$ curium $X$-rays were also seen. Figure 14 is an exanple of a standard gama-ray spectrum.

Since these $\mathrm{X}$-rays occur in a region of high Compton and $\mathrm{B}^{-}$ background, 1.e. In the region less than $\sim 200 \mathrm{keV}$, careful attention. was paid to correcting the observed count rates for a decaying background. This was accomplished using a program writen for a Texas Instruments desk calculator by Walter Loveland at Oregon State University. Basically, average backgrounds were calculated on the 
left and right-hand sides of the peak which were then used to calculate a background for the peak itself. A percentage error was also calculated to give an indication of the reliability of the results. This method proved to be highly satisfactory and gave quite reproducible resulcs. Once the decay rates were determined for both the $k_{a_{1}}$ and $k_{a_{2}}$ peaks in each spectrum, half-life analyses were carried out using several methods. Two computer codes, CLSQ 115 and FRANT IC, $116 \mathrm{a}, \mathrm{b}$ were employed. Due to the poor statistics from the low count rates, however, both these programs falled to resolve the $243_{\mathrm{Bk}}$ background. A more successful approach was reached by using a desk calculator for least square fitting and subtracting the long-lived components from the shorter components. While taking longer to complete, this method of "hand" analysis proved superior to existing computer codes since tt could be carrled out even with very low statistics. Once half-lives were established, it was a simple matter to determine $A_{0}$ values.

A number of considerations went into the cross-section calcularions. Besides the obvious correctlons for beam leve1, target thickness, detector efficiency, bombardment duration and chemical yield (determined by the growth of ${ }^{249} \mathrm{Cf}$ from ${ }^{249} \mathrm{Bk}$ tracer) a correction also needed to be applied to the $A_{0}$ value. The $A_{0}$ values obtalned were the sum of the $\mathrm{K}_{\mathrm{a}_{1}}$ and $\mathrm{K}_{\mathrm{a}_{2}}$ curium $\mathrm{X}$-rays. A correction had to be appliec 1 ) to account for those $K X$-rays not analyzed and; 2) to account for the fluorescence yieid $\left(\omega_{K}\right)$ of $K X$-rays from the curtum decay, Dittner and Bem1slo2 experimentally determined the relative transition probabilities of $k$ X-rays for curium which were found to be in excellent agreement with predicted values of Lu, Malik, and Carlson. 117 The experimental values 
were used to correct for the missing $k_{B_{1}}, K_{B_{2}}, k_{B}$, etc. X-rays. An estimace for the fluorescence yleld of $K X$-rays from curlum taken from Fink et a1.118 was $97 \%$.

\section{Americium/Curlum Samples}

A rather different approach was used in the analysts of data where the statistics were more reasonable, for example, in the cases involving amerlcium/curium samples. Here a peak finding program, SAMPO, 104 was used to locate photopeaks, calculate the $1 \mathrm{r}$ energy, and weasure their area. From the SAMPO analysis, the peaks were identified on the basis of half-life, energy, and the presence of sister peaks. The primary source for the peak Identification data was the recent summary of garra:ray Information by BInder ${ }^{19}$ et al. Cross-sections were then calcula d considering the percentage of the gamma-ray, length of bombardment, beam level, chentcal yleld, etc.

\section{Spontaneous Fission Data}

From the positions of the fission tracks, as recorded by the $X-Y$ plotter interfaced to the scanning microscope, plots were made of the number of tracks versus time (position). The time Intervals were calculated from the circumference of the drum and its revolution speed. In this way half-ilves could be determined for observed fission activities with half-lives within a range which depended upon the drum speed; that 1s, if the drum rotated very fast, then a spontaneous fission activity with a long half-life would have appeared as a unfform background activity whereab if the arum rotated too slowly, a short-lived activity would have decayed before reaching the wlca detectors. In cases where no 
actluity greater than background ( 1 track/mica) was seen, only half-lives between the limits due to the drum speed could be set. The activity would have to be very short- or very jong-lived not to he seen.

Cross-section calculations were corrected for total efficiency (see Section IV-D), beam intensity, length of bombardment, etc. In cases where no activities were observed only upper limits were obtained.

It must be kept in mind that the observance of a spontaneous fission activity in mica track detectors yields nothing more than half-life information. There is no mass nor $Z$ identification and, as will be shown later, simply assuming $z_{1}+z_{2}=z_{1+2}$ is not a sufficient dotermination of the $Z$ of the activity.

Estimates of limles to spontaneous fission branchlng ratios and thus of spontaneous fission half-lives were obtalned by assuming production cross-sections and half-lives for the activicies, calculating the spontaneous fission branch $\left(\underset{\sigma_{P R E D}}{\sigma_{S F}} \mathrm{SF}\right)$ and from that, the spontaneous fission half-life limit $\left(\frac{t_{2} \text { spred }}{S F \text { branch }}<t_{\frac{1}{2}} s F\right)$. The half-lives used for these calculations were obtained from the empirical estimates of alpha and electron capture half-lives of Kolesnikov and Demin, 41 of Taagepera and Nurmia, ${ }^{120}$ from the methods of Viola and Seaborg, 121 and of Keller and Müzel. 42 The margin for error here lo large, but should certainly be less than a factor of 5-10. The more dramatic errors are likely to be due to the assumed cross-sections. The cross-sections actually used rere reduced by factor of -15 from the calculated results (JORPLE). 122 : The basis for the cross-gection calculations and the 
rationalization for this reduction in cross-section will be explained In the next section.

\section{Cross-Section Estimates}

Estimates of the cross-sections for the formation of compound nuclei in heavy ion reactions are extremely helpful to the experimentalist. They enable cholces to be made regarding which target/projectile combinations to use, what ylelds to expect, what beam energles are optimut, etc. for the production of a particular 1sotope. These estimates can be particularly useful when there are little or no experiuental data in the region of Interest, as in the case of neutron deficlent actinide Isotopes.

The cross-section calculation code employed, JORPLE, developed by Jose Alonso 122,123 and J.O. Rasmussen 123,124 and based on a computer code of Sikkeland and Lebeck ${ }^{125}$, considers the formation of the compound nucleus followed by competition between fission and neutron emission as the major modes of deexcltation. Consideration is given to the Interaction potential, which is analogous to that of Thomas 126 and of Rasmussen et a1., 123,124 to angular momentum effects, to nuclear deformation, to fusion probability using the H111-Wheeler transmission formula, ${ }^{127}$ to compound nucleus formation using the formula of Blatt and Wefsskopf, ${ }^{128}$ and finally to deexcitation of the compound nucleus by neutron evaporation which is based on the Jackson mode1, ${ }^{129}$ and corrected for fission competition using the empirical formula of Sikkeland 130 for $r_{n} / r_{f}$, the ratio of neutron emission to fission. A detalled explanation of the code ay be found in the above references, 
espectally $122,123$.

As In all calculations of this sort, the cross-sections obtalned are quite sensitive to the parameters used such as $r_{0}$, (diffuseness), nuclear shape and $r_{n} / \Gamma_{f}$. The parametsrs employed in the calculations gave the best overall fits to the experimental data through 1973 for heavy Ion reactions for ions up to neon in this region of the table of 1sotopes. Th1s does not mean, however, that these parameters give best fits in isolated regions such as on the very neutron deflelent side of B-stability. Further, no consideration is given to evaporation of particles other than neutrons, although 1 t is generally assumed that neutron emission is greatly favored over that of charged particles in this 2 region. Neither are considerations applied to particular entrance channel problems of admittedly, perhaps, only a few specific projectiles, such as the break-up or dissociation of the projectile. Because these corrections are, in general, of little or no consequence, they are 1gnored. On the other hand, as In the case of the parameter values in Isolated or local cases, they may Indeed become important. The excitation functions calculated from this code agree extremely well In shape and energy with most of the experimental data in the actinide region. Unfortunately, it was discovered in this work that there are large discrepancies in cross-sections in the region of neutron deficient actinide 1sotopes as will be discussed at a later point. Figure 1 shows the calculated excitation function for the $11_{B}+235_{U}$ system. 


\section{A. $242 \mathrm{Bk}$}

Table 2 lists the varfous target/projectlle combinations and the beam energies used to produce ${ }^{242} \mathrm{Bk}$ as well as the (H.I.,pxn) and (H.I., $\alpha \times n$ ) products, curfum and: amerfclum, respectively. Each of the reactions used to produce ${ }^{24 \hat{2}} \mathrm{Bk}$ was run at least two times. This was easily possible since the bumbardments to produce this isotope were only $\sim 25$ minutes long. As many as 12 bombardments were carried out in ane 8 -hr shift. The experiments to produce the pxn and $\alpha \times n$ products were each run once for 5-6 hours in an effort to produce as much activity as possible.

Table 2

Sumary of Bombardments

\begin{tabular}{|c|c|c|c|}
\hline System & Reaction & Lab Energy (MeV) & Product (s) \\
\hline $10_{\mathrm{B}}+238_{\mathrm{U}}$ & $5 \pi, 4 \pi$ & 60 & $243,244 \mathrm{Bk}$ \\
\hline \multirow[t]{2}{*}{$10_{B}+238_{U}$} & $6 n$ & 67 & $242_{B k}$ \\
\hline & $p 7,5 n$ & 67 & $240,242 \mathrm{C}_{\text {叮 }}$ \\
\hline $10_{B}+235_{U}$ & $4 \pi$ & $52-60$ & none observed \\
\hline \multirow[t]{4}{*}{$11_{B}+235_{U}$} & $4 n$ & $55-65$ & ${ }^{242} \mathrm{BK}$ \\
\hline & $4 n$ & 60 & milked ${ }^{242} \mathrm{Cm}$ \\
\hline & p5, 3n & 60 & $240,242 \mathrm{~cm}$ \\
\hline & $a 4,3,2 n$ & 60 & $238,239,240_{\mathrm{Am}}$ \\
\hline \multirow[t]{3}{*}{$14 \mathrm{~N}+232 \mathrm{Th}$} & $4 n$ & 77 & $242_{\mathrm{Bk}}$ \\
\hline & p5,3n & 77 & $240,242 \mathrm{Cm}$ \\
\hline & $\alpha 2 \pi$ & 77 & 240 A] \\
\hline $15_{N}+232 \mathrm{Th}$ & $5 n$ & $76-93$ & $242 \mathrm{Bk}$ \\
\hline
\end{tabular}


The firet reaction, $238_{\mathrm{U}}\left(10_{\mathrm{B}, 4-5 n}\right)^{244,243_{B k}}$, was a test run for the Irradiation method, the counting system, and the chemistry. The energy was chosen to be close to the peak for the $5 n$ reaction to produce ${ }^{243} \mathrm{Bk}$. Since the energy used was actually on the low side of the 5n peak, a small amount of ${ }^{244} \mathrm{Bk}$ was also produced. Since the predominan ganmas enfted by ${ }^{243} \mathrm{Bk}$ and ${ }^{244} \mathrm{Bk}$ occur at energles greater than $200 \mathrm{KeV}$, their detection efficiency is much lower than that for the curium $x$-rays. Further, the absolute Inlensities are not known. Thus since ${ }^{243} \mathrm{Bk}$ and $24{ }^{4} \mathrm{Bk}$ have half-lives very close to one another, only a combined cross-section of $14 \mathrm{Hb}$ could be obtained from this experiment. This cross-section value is 30 times lower than that predicted by JORPLE. After double-checking the irradiation system and the chemistry to be sure the low cross-section was not due to experimental error, work then advanced to the production of ${ }^{242} \mathrm{BK}$. Evidence for a short-lived ( $<10 \mathrm{~m} / \mathrm{n}$ ) component in the curfur $\mathrm{k}_{\alpha_{1}}$, and $\mathrm{k}_{\mathrm{a}_{2}} \mathrm{x}-\mathrm{rays}$ was found $\mathrm{in}$ the first experiment, ${ }^{235_{U}}\left({ }^{11_{B}, x n}\right) 246-x_{B k}$, even though the time fron the end of bombardment to the detector was $\sim 25$ minutes. Subsequent bombardments using a shorter chemistry confirmed this component as having a half-life equal to $7.0 \pm 1.3 \mathrm{~m}$. A typical decay curve is shown in Figure 16 . The short-lived component stands out quite strongly and is easily resolved from the longer-lived activity of $243_{B k}$ (half-11fe $=4.5 \mathrm{hr}$ ). The excltation function for this activity from $11_{B}+235_{U}$ shown In Figure 17 agrees quite well in peak energy and width (FWhM $\approx 5 \mathrm{MeV}$ ) with the Alonso calculations for the 4 n reaction. The experimental cross-section of $\approx 10 \pm 2 \mu \mathrm{b}$, however, 1a approxinately a factor of 20 below that calculated. 
Since the peak energles for the $4 n$ and $5 n$ reactlons are calculated to fall wthin approximately $5 \mathrm{MeV}$, it was unclear whether this activity was due to ${ }^{241} \mathrm{Bk},{ }^{242} \mathrm{Bk}$, or both. An excitation function was, therefore, measured for any short-lived (half-life $\geq 3 \mathrm{~m}$ ) component in the curlum $\mathrm{X}$-rays produced by $10_{\mathrm{B}}$ on ${ }^{235} \mathrm{U}$. No evidence for a short-lived activity was found over the energy range considered (52-60 MeV). This energy range should have maximlzed production of ${ }^{241}$ Bk while that of ${ }^{242}$ Bk should have been at least an order of magnitude lower.

As a further check on the mass number and the cross-section for production of this 7 -alnute activity, ${ }^{232}$ Th was bombarded with $15 \mathrm{~N}$. The measured excitation function is shown in Figure 18. Once more, the experimental FWHM (10.1 HeV) and peak energy agree well with the calculations for the $5 n$ reaction, but the cross-section $(9 \pm 1 \mu b)$ is again $\sim 25$ times lower than predictions.

One of the best ways to confirm the mass number (and $z$ ) of an isotope is to positively identify its daughter in the original parent chemical fraction. While long-term alpha counting of the various berkelium fractions gave hints (1.e. 7 counts expected in 4 days, 6 seen) of the presence of ${ }^{242} \mathrm{Cm}$, the electron capture daughter of ${ }^{242} \mathrm{Bk}$, $1 \mathrm{t}$ was dec1ded to milk a number of separate bombardments for the ${ }^{242} \mathrm{Cm}$ daughter. A total of twelve 25 -minute bombardments was carried out using $11_{B}$ on $235_{v}$ at 59.5 MeV. Berkelium was Imediately separated ( 4.5 minutes from EOB) from the dissolved foll, and the final berkelium fractions from each run were pooled. A day or so later, ${ }^{244} \mathrm{Cm}$ tracer was added and a curium fraction was renoved from this combined berkeliun fraction. Since the 
half-ilfe of ${ }^{242} \mathrm{Cm}$ is 163 days, it was necessary to count for long periods of time. Figure 13 shows a 10-day a-spectrum of the final curium fräction. The ${ }^{242} \mathrm{Cm}$ peak stands out quite nicely as does the ${ }^{244} \mathrm{Cm}$. The ${ }^{241} \mathrm{Am}$ was used to calibrate the columns. From the amount of ${ }^{242} \mathrm{Cm}_{\mathrm{m}}$ present, a cross-section for ${ }^{242}$ Bk of $9.3 \pm 1.54 b$ was found, in excellent agreement with the direct measurement of $\mathrm{J} 0 \pm 2 \mu \mathrm{h}$.

It was conceivable that the $1 \mathrm{cw}$ cross-sections could be due to the decay of 242 Bk by alpha emission or even spontaneous fission. A number of berkelium fractions were alpha counted whthin 8 minutes for any short-lived activities. None were observed. In fact, no alphas at all were observed in the first few hours after bombardment! In order to void any losses due to chemical yleld and tine, a number of catcher foils were gamma and alpha counted approximately cre minute irom tie end of bombardment (EOB). As In the chemistry runs, no (unidentified) alphas were observed. (The main alpha peaks seen were due to $211_{\mathrm{At}}$, 211 Po (from EC decay of $211 \mathrm{At}$ ), $213 \mathrm{Fr}$ and $226_{\mathrm{Th}}$ ) No information was gained from direct gama counting of the catcher foils due to the Intense $B-, X-$, and $\gamma$-ray background in the foll.

That the Ilght berkelium 1sotopes do not decay by spontaneous fission has been shown by L.P. Somerville. 131 IIe bombarded urantum isotopes with $10_{B}$ and $11_{B}$ at various energles as part of a study of delayed fission. No unaccounced for fission activity was observed resulting in a spontaneous fission branch $<0.03 \%$ for ${ }^{242} \mathrm{Bk}$. Easthan and Grant 132 bowbarded ${ }^{232}$ Th with $14 \mathrm{~N}$ at the peak for the $4 \pi$ reaction and set a cross-section upper limit for the spontaneous fission of $24, \mathrm{Bk}$ at $50 \mathrm{mb}$ for a half-life greater than $2 \mathrm{~ns}$. 


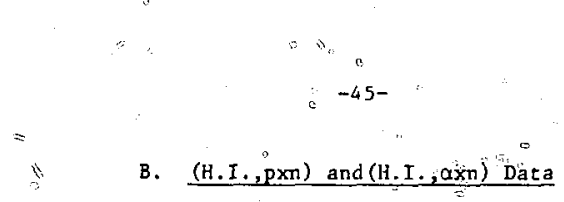

Since it now appeared that the cross-sections Eor the compound nucléus, $x n$ reactioñs, were indeed much lower than expleted, the question was raised as to what products were being produced. It was decided to examine the pxn and $\alpha \times n$ products, curlum and americium respectively, produced, directly in the same reactions and o, the same energies userd to produce ${ }^{242} \mathrm{Bk}$. A single bombardment resulting in $16.9 \mu \mathrm{A}-\mathrm{hrs}$ of $11 \mathrm{~B}^{+3}$ on $235 \mathrm{U}$ was carried out. Chemistry was performed to separate curium and americtum from the catcher foll." The resulting $\mathrm{Cm} / \mathrm{Am}$ fraction was both gama- and alpha-analyzed.

(3)

The results obtained are listed in Table 3 . The berkelium data were not determined in the same bombardment as the curium and́ americium but are given for comparison. After correcting the total yleld of $242 \mathrm{Cm}$ for the amount of $242 \mathrm{Bk}$ which decayed to ${ }^{242} \mathrm{Cm}$, there remains a considerable yleld of ${ }^{242} \mathrm{Cm}$ from other thais compound nucleus mechantsms. The total contribution of ${ }^{242}$ Am decay should be very small since the eaisston or transfer of only one alpha particle and no neutrons or other particles has a very small probability, especlally at an excitation energy of $\sim 43 \mathrm{MeV}$.

Data were also obtained from $10 \mathrm{~B}+238_{\mathrm{u}}, 14 \mathrm{~N}+232 \mathrm{Th}$, and $15 \mathrm{~N}+{ }^{235} \mathrm{U}$ as shown in Table 3. Only pxn information was gained In the $10_{B}=$ case since the $\alpha(1-3)$ n products were too long-11vêt co be seen. The cross-section for ${ }^{242} \mathrm{Bk}$ from ${ }^{10} \mathrm{~B}\left({ }^{238} \mathrm{U}, 6 n\right)^{242} \mathrm{Bk}$ was fourd to be $8.9 \pm 2.0 \mathrm{\mu b}$ and from ${ }^{14} \mathrm{~N}\left({ }^{232} \mathrm{Th}, 4 \mathrm{n}\right)^{242} \mathrm{Bk}$ to be $480^{3} \mathrm{nb}$. Only an upper $11 \mathrm{mit}$ to the compound nucleus cross-section for ${ }^{246}$ Es was determined due to 1 ts small cross-section and short half-11fe $(7.3 \mathrm{~m})$. 


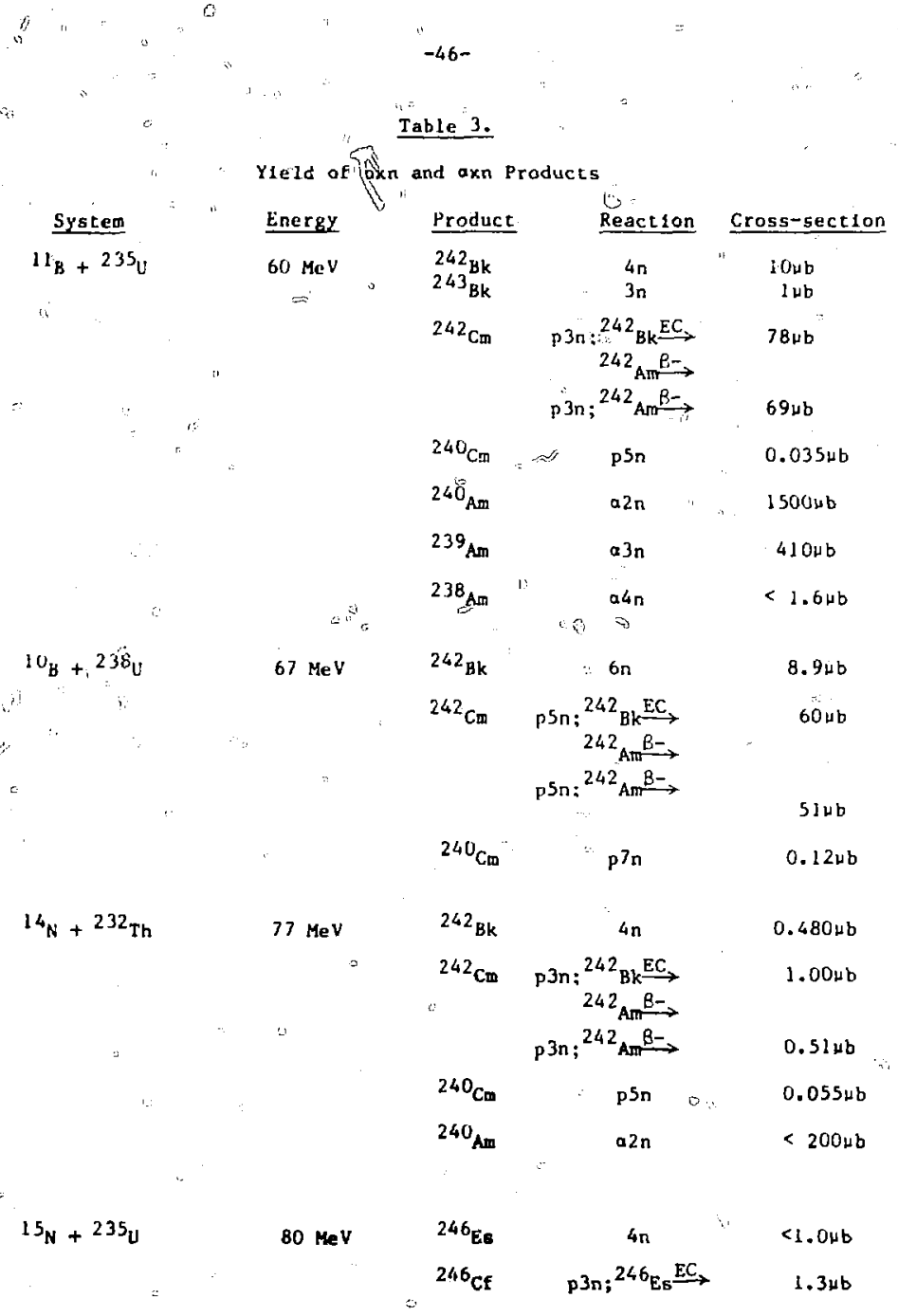




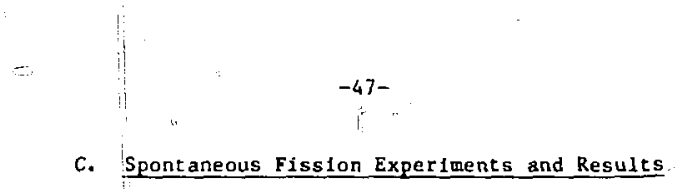

The bombardinents performed to search for spontaneous fission activity in even even neutron deficient actinide isotopes are listed in Table 4. No spontaneous fisston activities were seen with half-life 11mits less than approximately 15 hours and greater than several hundred nanoseconds in any of the bombardments. Using the method described previously, crude estimates were made for spontaneous fission branching ratios and spontaneous flssion half-1ives, shown also in Teble 4. The assumed cross-sections were taken to be about 15 times lower than those calculated by JORPLE to try to give a more realistic picture of the experimental cross-section for neutron deficient actinides. This factor was arrived at by comparing a number of different experimental cross-sections in this actinide mass regton with calculations.

\section{Table 4.}

Summary of Spontaneous Fission Bombardments

\begin{tabular}{|c|c|c|c|c|c|}
\hline Reaction & Is otope & ${ }^{0}$ calc ${ }^{\star}$ & $\%$ SF $F_{\text {EXPT }}$ & $T_{\frac{1}{2}{ }_{S F}}^{\dagger}$ EXPT & $\mathrm{T}_{1 / 2 \mathrm{SF}-\mathrm{PREI}}^{+}$ \\
\hline${ }^{3} \mathrm{He}+233 \mathrm{U}$ & $228_{\mathrm{Pu}}$ & $0 . \ln b$ & $<90 \%$ & $>2 \pi$ & $1.5 y$ \\
\hline${ }^{24} \mathrm{Mg}+{ }^{N A T}{ }^{1} L_{\mathrm{Pb}}$ & ${ }^{228} \mathrm{Pu}$ & $735 \mathrm{nb}$ & $<97$ & $>22 m$ & \\
\hline${ }^{3} \mathrm{He}+233 \mathrm{U}$ & $230_{\mathrm{Pu}}$ & $9 n b$ & $<5 \%$ & $>4 h r$ & $2.1 \times 10^{3} y$ \\
\hline${ }^{28} \mathrm{Si}_{1}+{ }^{208_{\mathrm{Pb}}}$ & ${ }^{232} \mathrm{Cm}$ & $15 \mathrm{nb}$ & $<30 \%$ & $>3.3 \mathrm{~m}$ & $28 m$ \\
\hline $40_{\mathrm{Ar}}+\mathrm{NAT}^{\prime} \mathrm{L}_{\mathrm{Hg}}$ & ${ }^{238} \mathrm{Cf}$ & $1.3 \mathrm{nb}$ & $<25 \%$ & $>4 \mathrm{sec}$ & $260 n s$ \\
\hline
\end{tabular}

* Calc. from JORPLE with corrections as described in text.

* Limite are based on the assumed cross-section and half-life.

+ Values obtained from Figure 3 . 


\section{DISCUSSION}

\section{A. Berkel1um-242}

From the data presented there seems little reason to doubt that the observed 7 -minute component of the curlum $X$-rays found in the berkelium chemical fraction is due to ${ }^{242} \mathrm{Bk}$. The most convincing evidence is the presence of ${ }^{242} \mathrm{Cos}$ in the berkelium chemical fraction. The only way it could be present is from the decay of the ${ }^{242}$ Bk parent. The shape of the excltation functions, the projectile energy for $\sigma_{\text {max }}$, the decay mode, the half -11fe value, and the cross-bombardments all lend additional support to the conclusion that this activity is indeed $242 \mathrm{Bk}$.

An important question 1 's: Is this the ground state of $242 \mathrm{Bk}$ or is it an isomeric state? Certainly an explanation of this activity as an isomer of berkelium would help explain the low cross-section (for this case). However, the experimental cross-sections for a number of neutron deficient Isotopes are also low compared with JORPLE calculations as shown in Table 5. Thus the low experimental cross-section does not appear to be out of line with other reported results. In order to examine the question of whethor or not this species is an isomerlc state, X-ray/gamma colncidence should be performed. This would reveal any correlations between gamma emission and the electron capture decay of the 1sotope, 1.e. whether or not the decay was to the ground state of curium. A major obstacle to this attempt is the low production cryss-section of the 1sotope. It is unlikely that enough activity can be produced to yleld any useful information, especially if chemistry must be performed. Perhaps the best possibility would exist with an on-line mass separator 
which could separate the berkellun and transport it to a colncidence detection system in a winimum amount of time. The limiting factor here would be the degree of separation and transportation efficiency.

Table 5.

\begin{tabular}{|c|c|c|c|}
\hline Reaction & gEX'I & of ORPLE & $\operatorname{Ref}$. \\
\hline${ }^{232} \operatorname{Th}\left(11_{B}, 5 n\right)^{238} \mathrm{Am}$ & $120 \mu \mathrm{b}$ & $2000 u b$ & 133 \\
\hline${ }^{232} \mathrm{Th}\left({ }^{12} \mathrm{C}, 4 \mathrm{n}\right){ }^{240} \mathrm{Cm}$ & $40 \mu \mathrm{b}$ & $185 \mathrm{Hb}$ & 134,135 \\
\hline 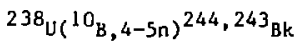 & $14 \mu \mathrm{b}$ & $414 \mathrm{Hb}$ & $\star$ \\
\hline $\left.238_{\mathrm{U}(} 11_{\mathrm{B}, 4 \pi}\right)^{245_{\mathrm{Bk}}}$ & $<20 \mu \mathrm{b}$ & $1200 \mu b$ & * \\
\hline${ }^{235} \mathrm{U}\left({ }^{\left.11_{B}, 4 \mathrm{n}\right)}{ }^{242} \mathrm{Bk}\right.$ & $10 \mu \mathrm{b}$ & $200 \mu \mathrm{b}$ & $\star$ \\
\hline${ }^{233} \mathrm{U}\left({ }^{12} \mathrm{C}, 5 \mathrm{n}\right)^{240} \mathrm{Cf}$ & $\sim 0.007 \mu \mathrm{b}$ & $0.150 \mu \mathrm{b}$ & 36 \\
\hline${ }^{23} 3_{\mathrm{U}}\left({ }^{12} \mathrm{C}, 4 \pi\right)^{241} \mathrm{CF}$ & $\sim 0.040 \mu \mathrm{b}$ & $1.4 \mu \mathrm{b}$ & 36 \\
\hline${ }^{234} \mathrm{U}\left({ }^{12} \mathrm{C}, 5 \mathrm{n}\right)^{241} \mathrm{Cf}$ & $\sim 0.015 \mu b$ & $0.740 \mu \mathrm{b}$ & 36 \\
\hline
\end{tabular}

It must be stressted that these disagreements are probably local perturbations and that, in general, JORHLE provides quite useful Information about actinide cross-sections.

* This work.

Another explanation for the low cross-section could lie in entrance channel effects, i.e. profectile break-up due to the Coulomb field of the target nuclei. While from this work there is no evidence for such a a problem, it is we $11-\mathrm{known}$ that both ${ }^{6} \mathrm{LI}$ and ${ }^{7} \mathrm{Li}$ are predisposed to break-up into ${ }^{3} \mathrm{He}, \mathrm{p} 2 \mathrm{n}$ and $\mathrm{a}, \mathrm{p} 2 \mathrm{n}$, respectively. ${ }^{136,137}$ Unternährer ${ }^{138}$ and co-workers have demonstrated ${ }^{9}$ Be break-up, and 011 erhead ${ }^{137}$ reported linited data on the breakmup of $10,11_{B}$. If dissociation of the projectile were to occur prior to the formation of the compound nucleus, the 
fusion yields would certainly be lower than expected. In fact, it has been found, both in this work and by others, 35 that fusion ylelds from boron reactions are uniformly lower than expected.

An additional contribution might also come from the fissionability of the target. Admittedly, ${ }^{232}$ Th and $238_{\mathrm{U}}$ are not considered very f issionable; however, ${ }^{233} \mathrm{U}$ and ${ }^{235} \mathrm{U}$ are. The compound nucleus yield would certainly be reduced if the target nuclel were to undergo fission prior tn or during fuston. This explanation seems unlikely, however, since the reactions ${ }^{234} \mathrm{U}\left({ }^{12} \mathrm{C}, 4 \mathrm{n}\right){ }^{242} \mathrm{Cf} ;{ }^{235} \mathrm{u}\left({ }^{12} \mathrm{C}, 5 \mathrm{n}\right){ }^{242} \mathrm{Cf}$; and

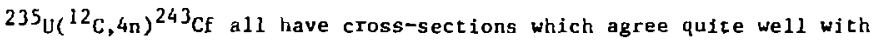
JORPLE calculat tons.

Assuring that the above mentioned contributions are small, the most likely explanation for the cross-section discrepancies lies in the calculations thenselves. As has been mentloned, the values used for such parameters $\rightarrow s, r_{0}$, etc. were determined from best $f$ th to the data avallable (1973) for the whole actinide region. It is quite possible that these parameters are not applicable in the neutron deficient region. The most likely parameter to be in error would be $\Gamma_{n} / \Gamma_{f}$. However, the change required if this is the only value in error is probably too great (lower by approxitately a factor of 3 ) and ylelds a somethat unrealistic $r_{n} / r_{f}$ rat10.8,139,140 More probably, swall changes in a number of parameters are required rather than a drastic change in any one.

\section{B. $(H, I,, p \times n)$ and $(H, I$, axn) Reaction Products}

In 1967 Fleury et al. 141,142 measured the production of ${ }^{240} \mathrm{C}_{m}$ and $242 \mathrm{~cm}$ from $10,11_{\mathrm{B}}$ on ${ }^{238} \mathrm{U}$. They interpreted the excitation functions 
to be due to a compound nucleus mechanism. Présumably, ${ }^{242} \mathrm{Bk}$ and $240_{\mathrm{Bk}}$ were produced which then decayed to their curlum daughters prior to the chemical separations. The cross-sections they reported were significantly higher than those measured in this work for ${ }^{242} \mathrm{Bk}$. In 1 ight of the newly weasured cross-sections for ${ }^{242} \mathrm{Bk}$, it did not seem reasonable that cross-sections as high as several hundred microbarns for ${ }^{242} \mathrm{Cm}$ could have come only from the decay of ${ }^{242} \mathrm{Bk}$ as assumed by Fleury. Indeed, this work has shown that such is not the case. Only a small contribution to the curium cross-section comes from the decay of berkellum. The primary yield of ${ }^{242} \mathrm{Cm}$ (and presumably ${ }^{240} \mathrm{Cm}$ ) has been shown to be due to either direct production of ${ }^{242} \mathrm{Cm}\left(240_{\mathrm{Cm}}\right)$ or to the evaporation of a proton and 3 (5) neutrons frot the crmpound nucleus. The latter seems extremely unlikely due to the high Coulomb barrier to charged particle emission from a nucleus with a $Z$ as high as 97.

This work further demonstrates the high ylelds of axn products in heavy ion reactions in the actinide region. The reaction showing the highest yield is the a2n followed by the a $3 n$. Again, it is unlikely that these products result from alpha evaporation, but rather, they are most probably the result of direct formation. To this effect, Hahn et al. 17 have demonstrated that axn products from ${ }^{12} \mathrm{C}+{ }^{239} \mathrm{Pu}$ are indeed the result of a transfer mechanism rather than a compound nucleus mechanisto They found, as well, that the $a 2,3 n$ product $y$ lelds (Cf) were much higher than the 2-4n compound nucleus yields ( $F_{m}$ ). Actually this is not too surprising since $\Gamma_{n} / T_{f}$ is so highly $z$ dependent. The (H.I.,pxn) and (H.I., axn) products have $Z$ 's lower than the $x$ products and, therefore, higher $\Gamma_{n} / \Gamma_{f}$ ratios. While nore compound nuclei may be formed Inttially, 
fewer of them are able to survive fission competition due to their lower $r_{n} / r_{f}$ ratios. Of particular note here is that the ylelds of the $\alpha 2,3 n$ products from ${ }^{12} \mathrm{C}+{ }^{239} \mathrm{Pu}$ are within a factor of $2-3$ of those reported for the compound nucleus reaction from ${ }^{12} \mathrm{C}+238_{U} 143$ producing the identical californfun isotopes! Kutzetsuv et al.144,145 also have reported axn reactions with cross-sections four times larger than the corresponding compound nucleus reaction using boron as the projectile; Bimbot et al. ${ }^{14}$ find significant axn yields with ${ }^{12} \mathrm{C}$ projectiles; and Brandshtetr et al. 146 find the a4n $\left({ }^{246} \mathrm{CF}\right)$ product from $16_{0}+238_{\mathrm{l}}$ to be equal in y leld to the $4 \mathrm{n}\left(246_{\mathrm{Cf}}\right)$ product from $12 \mathrm{C}+238 \mathrm{v}$.

The same trends were found in this work using boron and nitrogen projectiles on uranium and thorium targets. The compound nucleus crosssections are significantly lower than pxn and axn products from the same reaction. While no data were measured to confirm that these products result from direct reactions, it seens reasonable that such is the case. The particle "transferred" to the ${ }^{235} \mathrm{U}$ target in the boron bombardments co produce americium would be ${ }^{7} \mathrm{Li}$ followed by neutron emission. Hubert et al. 147 found very high yields of lithium transfer products in studies of boron on tantalum. $8_{B e}$ would be the particle transferred to form curium isotopes. As mentloned, Hahn 17 has studied ${ }^{8}$ Be transfers and found their ylelds to be significant.

These results suggest that, in some cases at least, it may be more profitable to attempt to produce a neutron deffcient actinide isotope via a transfer mechanism ( $p$ or $a x n$ ) than by a compound nusleus mechanism. Such ts the method used by Kutznetsov. 144,145 of perhaps even greater 
importance is the effect these data have on the " $z_{1}+z_{2}=$ New Element" hypothesis of Flerov and co-workers $148-154$ at Dubna. These researchers rely heavily upon spontaneous fission data for 1dentificaiton of new isotopes and elements whlch, as mentloned before, does not provide unambiguous proof of elther $Z$ or $A$ for a spontaneous fission activity. It has often kan claimed by this group in defense of their identification of various new elements and 1sotopes, that (H.I.,pxn) and (H.I., axn) reactions io not occur to any great degree $1 \mathrm{n}$ reglons of high $\mathrm{Z}$, and that, therefore, it is reasonable to assume the resulting spontaneous fission activicy is due to the compound nucleus, $1 . e \cdot, z_{1}+z_{2}=z_{1+2}$ * While $1 \mathrm{t}$ way be true that proton or alpha evaporation does not occur to any degree, direct transfer reactions giving the same products as proton or alpha evaporation reactions do occur and to a degree often in excess of the compound nucleus! Nor are excltation functions or angular distribution measurements necessarily sufficient for elimination of a transfer mechan1sm. For example, Fleury's 141,142 excitation function for ${ }^{238} \mathrm{U}\left({ }^{10} \mathrm{~B}, ?\right)^{242} \mathrm{Cm}$ looks deceptively like a compound nuc:.eus exc1tation function, and Hahn et al. 17 have cautioned that at certain bombarding energies it may not be posstble to distinguish the angular distributions of compound nucleus from transfer reactions.

\section{Spontaneous Fission Studies}

In the spontaneous fission experiments the only point of interest 1s that of californium. The pointe for ${ }^{228} \mathrm{Pu}, 230 \mathrm{Pu}$, and ${ }^{232} \mathrm{~cm} 11 \mathrm{e}$ below the predicted half-lives so no information was gained about their relation to these curves (FIgure 3). The number of beam particles 
necessary to ralse the limits determined for plutonium and curlum would require more bean time than is currently avallable for this rype of exper 1ment.

The lower limit to the spontaneous fission half-life for ${ }^{238} \mathrm{Cf}$ has been determined to be several seconds, while the extrapolated parabola ylelds a prediction of several hundred nanoseconds (Figure 3). The shortest electron capture half-life predicted for this isotope is 0.6 sec. 42 Therefore, if the spontaneous fission half-life were to follow these predictions and be several hundred nanoseconds, then the primary decay mode seen would be spontaneous fission. Since such is not the case, the spontaneous fission half-life is either not as short as predicted, or much, much shorter than predicted; or the alpha/electron capture half-life Is much shorter than predicted, l.e. quch shorter than the spontaneous fission half-life.

Undoubtedly, the straight extrapolation assumption is wuch too natve. Indeed, theoretical calculations of Viola and Wilkins 155 predict the spontaneous fission half-1lfe for ${ }^{238} \mathrm{CE}$ to be on the order of days! Their predictions are derived from experimental data using the liquid drop model, modifled to account for nuclear deformation and level siructure effects. Even-even isotopes were taken as reference nucle1. Their calculations show that fission 11 fetimes and barriers decrease regularly with increasing values of $z^{2} / A$, as predicted by the liquid drop model, but they do not decrease as dramatically as the empirical extrapolations in Figure 3 would predict. Deviations from thefr predictions are the result of irregularitles in nuclear structure caused by single particle effects, deformation, pairing interactions, etc. 
It is important to realize that, these results do not eliminate the possibility of the existence of spontaneous fission isomers of the Isotopes studied, which typically have half-lives shorter than several milliseconds, but probably only reflect the non-existence of spontaneous fission as the primary decay mode from the ground state within the limits determined.

While no conclusions can be reached regarding the true spontaneous fission half-lives of the isotopes examined, it seems clear that a simple extrapolation of the known points is not sufficient to predict spontaneous fission half-lives, a point of ten argued by the groups involved with the 260 J04 identification and half-1ife controversy. $150,156,157$ 
VIII. CONCLUSIONS

Evidence has been presented for the new berkelium 1sotope, ${ }^{242} \mathrm{Bk}$. originally it was hoped that ${ }^{241} \mathrm{Bk}$ and perhaps even $240_{\mathrm{Bk}}$ would also be found. Most probably, the combination of a short half-life and a small production cross-section prevented the observation in these Experiments of the lighter 1sotopes. Future experiments should focus on the shortest possible chemical separation--perhaps even on line-coupled with as sensitive an X-ray detection system as posstble. While the cross-section for ${ }^{242} \mathrm{Bk}(-10 \mu \mathrm{b})$ was small, it still should be possible to observe 241 Bk with the proper sensitivity.

A mafor problem would exist, however, if ${ }^{241}$ Bk decays by electron capture with a half-life very cloee to that of $242 \mathrm{~B}$. In that case, It may not be possible to resolve this isotope from ${ }^{242}$ Bk if 1 ts crosssection is less than several microbarns. In this sftuation, some sort of mass separation system, as mentioned earlier, would be appropriate. As before, the liniting factors would be cross-section and transportation/ separation efficiency.

of particular laportance is the result that ylelds of (Heavy Ion, $p \times n$ ) and (Heavy Ion, axn) transfer products are equal to or exceed the ylelds of the compound nucleus products in the actialde reglon. As has been emphasized, these findings point out the dangers in assuming yields of pxn and axn products to be negligible in the heavy element region. While charged particle evaporation may be miniagl in this $z$ region, one cannot neglect the production of the same products by a direct mechanisa. Defore new element or isotope may be clained, it must be 
shown that such directly produced products are not responsible for the new observations.

A systematic study of (H.I.,pxn) and (H.I.,axn) versus (H.I., $x_{n}$ ) reactions should be performed to further examine this phenomerion. Projectiles and targets should be varied to look for mass effects, evenmodd effects, $z$ effects. Heavier projectiles should be used to examine the difference between them and 11 ghter projectiles.

As the $Z$ of the products increases, the experiments become increasingly more difficult.: The cross-sections are dramatically lower for the production of, for example, fermium compared to curlum. Further, the half-lives of the most interesting products are on the order of minutes rather than hours or days making chemlcal seaprations, especially awong the actinide elements, very difficult. Examples of systems that might provide useful information would be:

$$
\begin{aligned}
& 18_{0}+{ }^{232} \text { Th , } \quad 250_{\text {Cf }} \ldots \times n \\
& 249_{B k} \star \ldots \times n(+p) \\
& { }^{12} \mathrm{C}+{ }^{238_{U}(4)} \quad{ }^{246} \mathrm{C}^{*} \ldots \times \mathrm{xn}(+\infty) \\
& 15_{\mathrm{N}}+{ }^{238} \mathrm{U} \quad 253_{\mathrm{Es}} \star \ldots \times \mathrm{xn} \\
& { }^{252} \mathrm{Cf} \ldots \ldots \times n(+\mathrm{p}) \\
& 249_{B k} * \ldots \times n \text { (ta) }
\end{aligned}
$$

Once a systeatic study of the actinides has been completed, the study could be expanded to include the region around lead where fission is no longer eliuinating wost of the products.

(1) Examined in part by Hahn et al.17 
While the lack of observations in the spontaneous fission experinents was at first disappointing, it was not too surprising. The sensitivity for three of the lsotopes was insufficient to draw any conclusions, while the results for ${ }^{238}$ Cf do not rule out a very, very short half-life $(<\sim 100 \mathrm{~ns})$. What is of importance, however, 15 the fact that the half-lives do not appear to follow strictly empirical predictions on the neution deficlent side of the 152-neutron subshell." Future experiments should concentrate on the higher $Z$ elements since these have the greatest possibility of being observed, 1.e., require less bean intensity for the experiments. The drum speed should be increased as much as possible to search for extremely short half-lives. Decay-in-flight techniques could be used to reach even shorter half-lives. Unfortunately, however, If a short-lived activity is found, there is no simple way using the present methods to distingulsh it from an isomer of the same or even another element. As has been mentioned time and again, spontaneous fIssion measurements alone are Inconclusive regarding the $Z$ and mass of an activity.

In conclusion then, this work has pointed out the grave errors in assuming the identity of a new element or sotepe based solely on the assumption that $z_{1}+z_{2}=z_{1+2}$, especially when spontaneous fission fs the only method of Identification. It further cautions those scientists who doggedly insist on sticking to empirical systematics that such predictions, while of ten quite useful, are hot infallible and are at times subject to question. 


\section{REFERENCES}

1. J.M. Alexander, G.N. S1monoff, Phys. Rev. 133, B93-B103 (1964).

2. V.E. Viola, T. Sikkeland, Phys. Rev. 128, 767-774 (1962).

3. M. Lefort, Rep. Prog. Phis. 39, 129-173 (1976).

4. G. Friedlander, J. Kennedy, J.M. Miller, Nuclear and Radiochemist iy, 2nd ed., John Wiley and Sons, Inc., NY, p. 302 (1964).

5. R. Vandenbosch, J.R. Huizenga, Nuclear Fission, Academic Press, NY, p. $227-253$ (1973).

6. K. Vandenbosch, J.R. Hulzenga, Proc. IntI. Conf. Peaceful Uśes At. Energy, 2nd 15, p. 284, Uniced Nations, Geneva (1958).

7. H. Delagrange, S.Y. LIn, A. Fleury, J.M. Alexander, Phys. Rev. Lett. $39,867-870$ (1977).

8. J. Gilat, A. Fleury, H, Delagrange, J.M. Alexander, Phys. Rev." C16, 694-705 (1977).

9. J. W1lczynsk1, J. Nuc1. Phys. A216, 386-394 (1973).

10. D.H.E. Gross, H. Kalinowski, Phys. Lett. 48B, 302 (1974).

11. M. Lefort, Y. LeBeyec, J. Peter, "On the Fusion R̂eactions Betwén Complex Nucle1" XI Winter Meeting on Nuclear Physics (January 31973 ).

12. T. S1kkeland, Phys * Lett., 27B, 277-279 (1968).

13. P.M. Strudler, 1.L. Preiss, R. Wolfgang, Phys. Rev. 154, 1'26-1135 (1967).

14. R. Bimbot, D. Gardes, M.F. Rfivet, Nucl. Phys. Al89, 193-219 (1972).

15. R. Btmbat, D. Gardes, R.L. Hainn, Y. deMoras, M.F. Rivet, Nucl. Phys. A228, 85-111 (1974).

16. R. Bimbat, D. Gardes, M.F. RIvet, Phys. Rev. C4, 2180-2184 (1971). 
$c=\quad \therefore \quad \therefore \quad-58-$

While the lack of observations in the spontaneous fission experiments was at first disappointing, it was not too surprising. The sensitivity for three of the 1sotopes was insufficlent to draw any conclusions,' while the results for ${ }^{238} \mathrm{Cf}$ do not rule out a very, very short half-life (< $100 \mathrm{~ns}$ ): What $1 \mathrm{~s}$ of 1mportance, however, is the fact that the half-lives do not appear to follow strictly emplrical predictions on the neutron deficient side of the 152-neutron subshe11. Future expertonts should concentrate on the higher $z$ elements since these have the greatest si possibility of befig observed, 1.e., require less beam intensity for the expertinents. "The drum speed should be increased as much as possible to search for extremely short half-lives. Decay-in-flight techniques could be used to reach even shorter half-lives. Unfortunately, however, If a short-lived activity is found, there is no simple way using the present methods to distinguish it from an isomer of the same or even is

another element. As whas been mentioned time and again, spontaneous fission measurements alone are inconclusive regarding the $z$ and mass of an activity.

In conclusion then, this work has pointed out the grave errors in assuoing the Identity of a new element or 1sotope based solely on the assumption that $z_{1}+z_{2}-z_{1+2}$, especially when spontaneous fission is the oniy wethod of Identification. It further cautions those scientists who doggedly Insist on sticking to empirical systematics that such predictions, while of ten quite useful, are not infallible and are at จ times subject to question. 


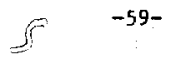

REFERENCES

1. J.M. Alexander, G.N. Simonoff, Phys, Pev. 133, B93-B103 (1964).

2. V.E. Viola, T. Sikkeland, Phys. Fey. 128, 767-774 (1962).

3. M. Lefort, Rep. Prog. Phys. $39,4 y-173$ (1976).

4. G. Eriedlander, J. Kenneciy, J.M. Miller, Nuclear and Radiochemistry, 2nd ed., John wiley and Sons, Inc., NY, p. 302 (1964).

5. R. Vandenbosch, J.R. Hulzenga, Nuclear Fission, Academic Press, NY, p. 227-253 (1973).

6. K. Vandenbosch, J.R. Hulzenga, Proc. Intl. Conf. Peaceful Uses At. Energy, 2nd 15, p. 284, United Nat1ons, Geneva (1958).

7. H. Delagrange, S.Y. Lin, A. Fleury, J.M. Alexander, Phys. Rev. Lett. 39, 867-870 (1977).

8. J. Gilat, A. Fleury, H. Delagrange, J.M. Alexander, Phys. Rev. C16, 694-705 (1977).

9. J. W1 lczynsk1, J. Nuc1. Phys. A216, 386-394 (1973).

10. D.H.E. Gross, H. Kallnowsk1, Phys. Lett. 48B, 302 (1974).

11. M. Lefort, Y. LeBeyec, J. Peter, "On the Fusion Reactions Between Complex NucleI" XI Winter Meeting on Nuclear Physics (January, 1973).

12. T. Sikkeland, Phys. Lett., 27B, 277-279 (1968).

13. P.M. Strudler, I.L. Preiss, R. Wolfgang, Phys. Rev. 154, 1126-1135 (1967).

14. R. Bimbot, D. Gardes, M.F. Rivet, Nuc1. Phys. Al89, 193-219 (1972).

15. R. Bimbot, D. Gardes, R.L. Hahn, Y. deMoras, M.F. Rtvet, Nucl. Phys. A228, 85-111 (1974).

16. R. Blmbot, D. Gardes, M.F. Rfuet, Phys, Rev. C4, 2180-2184 (1971). 
17. R.L. Hahn, P.F. Ditcrer, K.S. Toth, O.L. Keller, Phys. Rev. Clo, $1889-1903(1974)$.

18. W.R. Phillips, Rep. Prog., Phy6. 40, 345-409 (1977).

19. J. Galin, B. Gatty, M. Lefort, J. Peter, X. Tarrango, R. Basile, Phys. Rev. 182, 1267-1276 (1969).

20. J. Galin, D. Guerreau, M. Lefort,"J. Peter, X. Tarrango, R. Basile,' Nuc 1. Phys. A159, 461-480 (1970).

21. A. Ghiorso, M. Nurmia; K. Eskola, J. Harris, P. Eskola, Phys. Rev. Lett. 24, 1498-1503 (1970).

22. G.H. Flerov, Y.T. Oganesyan, Y.V. Lobanov, Y.A. Lasarev, S.P. Tretlakova, I.V. Kolesov, V.H. Plotko, Nucl. Phys. Al60, $181-192(1971)$.

23a. C.E. Bemis, Jr. and J.R. Nix, Comments on Nuciear Particle Physics $\geq 65(1977)$.

23b. Proceedings of the International Symposium on Superheavy Elements, Lubbock, Texas, March 9-11, 1978.

23c. R.J. Otto, D.J. Morrissey, D.M. Lee, A. Ghiorso, J.M. Nitschke, G.T. Seaborg, M.M. Fowler, R.J. Silva, Lawrence Berkeley Laboratory Report LBL-6509 (1977).

24. R.J. Otto, A. Ghiorso, D.M. Lee, R.E. Leber, S, Yashita, G.T. Seabarg, Lawrence Berkeley Laboratory Report LBL-7104 (1977).

25. R.J. Otto, D.J. Horrissey, G.T. Seaborg, W.D. Loveland, Lawrence Berkeley Laboratory Report 1,BL-7710 (1978).

26. W.J. Swiuteck1, "Prospects for Superheavy Mucle1" Nuclear Reactions Induced by Heayy Ions, Narth-Holland, p. 779-747 (1970).

27. J.M. Nitochke, Lawrence Berkeley Loboratory Report LHL-7705 (1978). 
28. A.G. Artyukh, V.V. Volkov, G.F. Gridnev, A.S. Il'inov, V.L. Mrkheev, Yad F12. 19, 54-61 (1974).

29. I. Zvara, Jo1nt Institute for Nuclear Research, Dubna, JINR-E12-7547 (1973).

30. G.N. Flerov, Y.T. Oganesyan, Y.V. Lobanov, A.A. Pleve, C.M. Ter-Akop'yan, A.G. Demin, V.I. Chepugin, Y.P. Tretyakov, Joint Institute for Nuclear Research, Dubna, JINR-P7-7409 (1973).

31. Y.T. Oganesyan, H. Bruchertseifer, G.V. Buklanov, V.I. Chep1g1n, C.V. Sek, B. Erchler, K.A. Gavrilov, H. Caeggeler, Y.S. KorotkIn, O.A. Orlova, T. Reetz, W. Seidel, G.M. Ter-Akop'yan, S.P. Tretyakova, 1. Zuara, Nucl. Phys, A294, 213-224 (1978).

32. S.G. Thompson, A. Ghiorso, G.T. Seaborg, Phys. Rev. $\underline{80}, 781-789$ (1950).

33. K. Holf, J. Unik, Phys, Lett. 38B, 405-407 (1972).

34. A. Chetham-Strode, Jr., Lawrence Berkeley Laboratory Report UCRL-3322 (Ph.D. ThesIs) (1956).

35. R.L. Hahn, (ORNL) private communication.

36. R.J. Silva, R.L. Hahn, K.S. Toth, M.L. MaIlory, C.E. Bemis, Jr., P.F. Dittner, O.L. Keller, Phys. Kev. C2, 1948-1951 (1970).

37. V.L. Mikheev, V.I. Ilyushchenko, M. B. Miller, J. Nuc1. Phys. (USSR) $2,49-55(1967)$.

38. T. Sikkeland, A. Shiorso, Phys. Lett. 24B, 331-332 (1967).

39. P. Eskola, K. Eskrila, M. Nurnia, A. Chiorso, Lawrence Berkeley Laboratory Report, LBL-2315 (i972).

40. B.G. Sarvey, G. Herrmann, R.W. Hoff, D.C. Hoffman, E.K. Hyde, J.J. Katz, O.L. Keller, M. Lefort, G.T. Seaborg, Science 193, 
$127 i-1272(1976)$.

41. N.N. Kolesnikov, A.G. Demin, Joint Institute for Nuclear Research, Dubna, JINR-P6-9420 (1975)

$$
\text { JINR-P6-9421 (1975). }
$$

42. K.A. Keller, H. Müze1, Nucl Phys. Al48, 615-624 (1970).

43. Data from LBL Table of Isptopes group (to be published as Table of Isotopes, $7 \mathrm{th}$ ed.)

44a. S. Bjornhola, J. Borggreen, E.K. Hyde, Nucl. Phys Al56, 561-569 $(1970)$.

4db. S.G. Nilsson, C.F. Tsang, A. Sobiczewski, 2. Syzmansk1, S. Wychech, C. Gustafson, I.L. Lam, P. Mo11er, B. Nilsson, Nucl Phys. Al31 $1-66(1969)$.

44c. V.M. Strutinsky, Nuc1. Phys, A95, 420-444 (1967)

Nuc 1. Phys. A122, 1-33 (1968).

45. L. Yaffe, Ann. Rev. Nuc1. Sc1. 12, 153-188 (1962).

46. R.L. Blanchard, B. Kahn, and R.D. Blrkhoff, Health Phys. 2 , $246-255(1960)$

47. 0.C. Aumann, and G. Müllen, Nuel. Inst. and Meth. 115, 75-81 (1974).

48. G. Müllen, and D.C. Aumann, Nuc1. Inst. Meth. 128, 425-428 (1975).

49. Yu. S. Korotkin, Joint Inst1tute for Nuclear Research, Dubna, JINR-P6-7400 (1973).

50. J.E. Evans, R.W. Lougheed, M.S. Coops, R.W. Hoff, and E.K. Hulet, Lawrence Berkeley Laboratory Report UCRL-73121 (CONF 711002-1) (1971).

51. T. Suzuk1, S. Baba, H. Umezawa, and H. Amano, Nucl. Inst. Me th. 87. $311-312(1970)$. 
52. J. Buch, N.I. Balalykin, A.V. Skrypnik, V.V. Katrasev, JoInt Inst1tute for Nuclear Research, Dubna, JINR-P]3-9783 (1976).

53. W. Parker, Methods in the Prepraration of Radloactive Material, Göteborg, Elanders Boktrycker1 Aktiebolag (1965).

54. W. Parker, and W. Gullholmer, Proc. of the Seminar on the Preparation and Standardization of Isotope Targets and Fol1s, AERE-R 5097, Paper $10(1965)$.

55. V. Verdinger, Proc. of the Seminar on the Preparation and Standardization of Isotope Targets and Folls, AERE-R 5097 Paper 12 (1965).

56. K.M. Glover, P.S. Robinson, Proc. of the Seminar on the Preparation and Standardization of Isotope Targets and Folls, AERE-R-5097 Paper 11 (1965).

57. M. Dakowsk1, H. Plekarz, M. Sawinsk1, Inst. of Nuc1. Res. Rept. No. 595/1A/PL, Warsaw (1965).

58. D.J. Carswell, and J. Milsted, J. Nucl. Energy I, 4, 55-54 (1957).

59. E. Bruninx, G. Rudstam, Nucl. Inst. Meth. 13, p. 131-140 (1961).

60. V.A. Gorodysk11, Yu. F. Rowanov, A.V. Sorok1na, M.I. Yakunin, PTE, $\underline{5}, 128-130$ (i959).

61. K.E. Williams, R.E. Leber, C. Lee, Lawrence Berkeley Laboratory Report LBL-6584 (1978).

62. Maynard Mfchel, (LBL) private communication.

63. L.C. Northcliffe, and R.F. Schlllirig, Nuclear Data Tables $\underline{7}, 223-463$ (1970).

64. R.A. Gough, R. Lam, C. Kartinez, D. Morris, Nucl. Inst. and Meth. 138, 415-419 (1976). 
65. L.B. Magnussun, and M.L. Anderson, J. Am. Chem. Soc. 76, 6207 (1954).

66. K. Street, S.G. Thompson, G. T. Seaborg, J. Am. Chem. Soc. 22 , $4832-4835(1950)$.

67. K. Street, G.T. Seaborg, J. Am. Chem. Soc. 22, 2790-2792 (1950).

68. S.G. Thompson, B.G. Harvey, G.R. Choppin, G.T. Seaborg, J. Aro. Cher. Soc. $76,6229-6236(1954)$.

69. D.F. Peppard, J.W. Mol 1ne, G.W. Mason, J. Inorg. Nucl. Chem. $\underline{4}$, $344-348(1957)$.

70. J.B. Knauer, and B. Weaver, Oak Ridge Hational Laboratory Report ORNL-TM-2428 (1968).

71. F.L. Moore, Anal. Chem. 38, 1872-1876 (1966).

72. R.M. Dlamond, K.S. Street, Jr., C.T. Seaborg, J. Am. Chem. Soc. I6, $1461-1469(1954)$

73. G.K. Choppin, B.G. Harvey, S.G. Thompson, J. Inorg. Nucl. Chem. 2 , 66-68 (1956).

74. K.F. Buchanan, J.P. Far1s, K.A. Orland1n1, J.P. Hughes, TIL-7560, $76(1958)$.

75. L.R. Bunney, N.E. Ballou, J. Pascual, S. Fot1, USNRDL-TR-228 (1958).

76. J. Goffart, and G. Duyckaerts, Anal. Chtm. Acta. 46, 91-99 (1969).

77. D.F. Peppard, P.R. Gray, U.S. Patent 2,683,655 July 13, 1954.

78. J.J. Katz, and G.T. Seaborg, The Chem1stry of the Act Intde Elements, John Wiley and Sons, New York (1957).

79. Cornelius Keller. The Cheaistry of the Transuranium Elements, Verlag Chevie GobH, Weinhein; Geraany (1971). 
80. G.H. Higgins, The Radiachemistry of the Transcuruiug Elements, Subcommitcee on Radlóchemistry, Nat' 1 Acad. Sclence - Nat'1. Regearch Counc11 (1960).

81. R.A. Pennewan, and T.K. Keenan, The Radfochemistry of Americium and Curium, Subcommittee on Radiochemistry, Nat'1. Acad. Sc1. Nat'1 Research Council (1960).

82. D.F. Peppard, Ann. Rev, Mucl. Sc1. 21, 365-396 (1971).

83. C.T. Seaborg, "ActInldes and Transactinldes," KIrk-0thmer: Encyclopedfa of Chem. Tech. Vol. 1, 3rd ed., 456-481, John Wiley and Sons, Inc. (1978).

84. E.K. Hyde, I. Perlman, G.T. Seaborg, The Nuclear Properties of the Heavy Elements, Vol. 2, Bover Publications, New York (1971).

85. V.N. Kosyakov, N.G. Yakovlev, G.M. Kazakova, E.A. Erin, and V.V. Kopytov, Rad lokh1⿴囗十) ya 19 (4) 486-489 (1977).

86. E.G. Chudinov, V.N. Kosyakov, I.K. Shvetsov, Yu. I. VereshchaguIn, Transplutiontum Elements, ed. W. Miller and R. Lindner, p. 49-57, North-Holland Publ. Co., Amsterdam (1976),

67. B.F. Myasoedov, J. Inorg. ivuc I. Chen., Supplement, p. 151-155, (1956).

88. V.N. Kasyokov, E.G. Chudinov, and I.K. Shvetsov, J. Inorg. Nuc 1. Chen., Supplewent, p. 99-103 (1976).

89. G.M. Kazakova, V.N. Kosyzkov, E.A. Erin, J. Inorg. Nucl. Chem. Supplement, 105-107 (1976).

90. S.G. Thompson, A. Ghiorso, C.T. Seaborg, Phy. Rev. 80, 781-789 (1950). 
91. J. Koo1, R. Boden, J. W1zkst1a, J. Inorg. Mucl. Chem. 26, 2300-2302(1964).

92. D.F. Peppard, S.W. Mollne, G.W. Mason, J. Inorg. Nucl. Chem. $4,344-348(1957)$.

93. E.S. Gureev, V.K. Kosyakoy, G.N. Yakovlev, Radiokhimiya 6., $655-665(1964)$.

94. E.P. Horowitz, C.A.A. Bloomquist, D.J. Henderson, J. Inorg. Nuc1. Chem. 31, 1149-1166 (1969).

95. E.P. Horowitz, C.A.A. Bloomquist, D.J. Henderson, D.E. NeIson, J. Inorg. Nacl. Chem. 31, 3255-3271 (1969).

96. J.C. Stewart, H.W. Crandall, J. Amer. Chem. Soc. 73, 1377-1378 (1951).

97. D. F. Peppard, G.W. Mason, J.L. MaIer, W.J. Driscoll, J. Inorg. Nuc 1. Chem. 4, 334-343 (1957).

98. H.J. MeDowe11, P.T. Perdue, G.K. Case, J. Inorg. Nuc1. Chem 38, $2127-2129(1976)$.

99. Helen Sm1th, Los Alamos Sctentific Laboratory Report LA-1721, 4ch ed. (1976).

100. A.M. Poskanzer, B.M. Foreman, Jr., J. Inorg. Nucl, Chem. 16. $323-336(1961)$.

101. P.A. Baisden, D.M. Lee, Lawrence Berkeley Loboratory Report LBL-7741 (1978).

102. P.F. Dittner, C.E. Bem1s, Jr., Phys. Rev. A5, 481-484 (1972).

103. Diana M. Lee, (LBL) private coamunication.

104. J.I. Routt, and S.G. Prussin, Mucl. Inst Heth. 72, 125-142 (1969). 
105. P.B. Price, R.M. Walker, J. Appl. Phys, 33, 3400-3406 (1962).

106. E.C.H. S11k, R.S. Barnes, Ph11. Mag. 4, 970 (1959).

107. P.B. Price, R.M. Walker, J. Appl. Phys, 33, 2625-2628 (1962).

108. R.L. Fle1fcher, P.B. Price, R.H. Walker, E.L. Hubbard, Phys, Rev. 133 A1443-A1449(1964).

109. P.B. Price, R.L. Fle1scher, Ann. Rev. Mucl. Sc1. 21, p. 295-334 (1971).

110. P.B. Price, R.M. Walker, J. Appl. Phys. 33, 3407-3412 (1962).

111. R.L. Fleischer, P.B. Price, R.M. Walker, Nuclear Tracks in

Solids - Principles and Applications, University of California Press (1975) Berkeley.

112. Am. Soc, for Testing Mater1als, Supplenent to Book of ASTM Standards Part II, 1042-1050 (3962).

113. L. Kleppe, and Sister Mary Rogers, R.S.M., Lawrence Berkeley Laboratory Report UCRL-17075 (1966).

114. M. Murmia, T. Sikkkoland, R. Silva, A. Chiorso, Phys, Lett. 26B, 78-80 (1967).

115. J.B. Cumming, Applications of Computers to Nuclear and Kadiochemistry NAS-NS 3107 P. 25-33 (1962).

116a. J.F. W11d, (LLL) private comentcation.

116b. P.C. Rogers, Tech. Rpt. 76 (NY0-2303) MIT, (1962).

117. C.C. Lu, F.B. Mal1k, T.A. Carleon, Mucl. Phys. Al75, 289-299 (1971).

118. R.W. F1nk, R.C. Jopson, Hans Mark, C.D. Swift, Reviewn of Hodern Phyelcs $38,513-540(1966)$.

119. I. Binder, R. Kraus, R. Kiefn, D. Lee, M. Fowler, Lawrence Berkeley 
Laboratory Report LBL-6515 (1977).

120. R. Tagepera, M. Murn1a, Ann. Ac. Sc1. Fennicae AVI-78 (1961).

121. V. Vicla, and G.T. Seaborg, J. Inorg. Nucl. Chem. 28, 741-761 (1966).

122. J.R. Alonso, Gaelln, Handbuch der Anorganischen Chemle, Band Fb, Te11 A1. 28 (1973).

123. J. Alonso, J,0. Rasaussen, Bull. Am. Phys. Soc. 17, 78 (1972).

124. J.0. Rasmussen, Sugawara-Tanabe, Nuc1. Phys. Al71, 497-521 (1971).

125. T. Sikkeland, D.F. Lebeck, (unpublished).

126. T.D. Thomas, Phys. Rev. 116, 703-712 (1959).

127. D.L. H111, J.A. Wheeler, Phys. Rev. 89, 1102-1145 (1953).

128. J.M. Blatt, V.F. Welsskopf, Theoret1cal Nuclear Physics, New York, p. $319(1966)$.

129. J.D. Jackson, Can. J. Phys. 34, 767 (1956).

130. T. S1kkeland, A. Ghiorso, M. Nurmia, Phys. Rev. 172, 1232-1238 (1968).

131. Lo, Sowerville, (LBL) unpublished data.

132. D.A. Easthas, I.S. Grant, J. Phys. (Par1s) Colloq. 5, 9 (1972).

133. Kenneth E. Thoses III, (LBL) unpublished daca.

134. L.1. Guseva, B.F. Myasoedov, N.I. Tarantin, K.V. Filippova, Sov. Phys. JETP 10 694-696 (1960).

135. Kiaberly E. Williams, (LBL) unpubItshed data.

136. K.0. Pfe1ffer, E. Speth, K. Bethge, Nucl. Phys. A206, 545-557 (1973).

137. R.W. Ollerhead, C. Chasuan, D.A. Bromley, Phys. Rev. 134, B74-B89 (1964). 
138. J. Unternährer, J. Lang, R. Mfller, Phys. Rev.' Lett, 40, 1077-1079 (1978).

139. A. 'Gavron, H.C. Brite, E. Konecny, J. Weber, J.B. Withelmy, Phys. Rev. C 13, 2374-2384 (1976).

140. A. Gavron, H,C. Britt, P.D. Goldstone, R. Schoenwackers, J. Weber, J.B. Wilhelmy, Phys. Rev. C 15, 2238-2240 (1977).

141. A. Fleury, F. Mivielle, G.N. Simonoff, Phys, Lett. 24B, 576-577 : (1967).

142. A. Fleury, F. Hubert, J. de Physique 10, 855-868 (1970).

143. T. Sikkeland, J. Maly, D.F. Lebeck, Phys. Rev. 169, 1000-1006 (1968).

144. V.I. Kuznetsov, K.K. Skobelev, G.N. Flerov, Sov. J. Muc1. Phys. 5, 191-192(1967).

145. V.I. Kuznetsov, N.K. Skobelev, G.N. Flerov, Sov. J. Muc1. Phys. 4, 70-71 (1966).

146. I. Brandshtetr, V.V. Volkov, V.A. Ermakov, T.S. Zvarova, M. Krzh1vanek, Y. Haly, S. Hug-Kue1, Radiokhimiya 5, 706-711 (1963).

147. F. Hubert, H. Delagrange, A. Fleury, Mucl. Phys. A228, 415-431 (1974).

148. G.N. Flerov, V.A. Karnaukhov, Jolnt Instltute for Nuclear Research, Dubna, JINR-D-1798 (1966).

149. G.K. Flerov, Joint Institute for Huclear Research, Dubna, JINR-E7-4207 (1968).

150. V.A. Druin, Ruclear Regetions Induced by Heevy Ions, Heidleberg North-Holland, P. 657-665 (1970). 


$$
=-70-
$$

b)

151. I. Zvara, Jo1nt Institute for Nuclear Research, Dubna, J1NR-E7-7547 (1973).

152. Y.T. Oganesyan, Y.P."Tret'yakov, A.S. Il'1nov, JINR-D7-8099 (1974).

153. Y.T. Oganesyan, "Fusion and Fission Induced by. Heavy Ions," Heidleber $\mathrm{B}(1974)$.

154. Y.T. Oganesyan, Joint Institute, for Nuclear Research, Dubna, J1NR-P7-9503 (1976).

155. V.E. Viola, B.D. Wilk1ns, Mucl. Phys. 82, 65-90 (1966).

156. A. Ghiorso, Lawrence Berkeley Laboratory Report LBL-5037 (1976).

157. V.A. Druin, Joint Institute for Nuclear Research, Dubna, JINR-E7-9546 (1976). 


\section{LIST OF FIGURES}

F1g. 1. Calculated Sxcitation func ion for $11 \mathrm{~B}+235_{\mathrm{U}}$ (reference 122 ).

F1g. 2, Chart of the nuclides showing the transuranium lisotopes.

F1g. 3. Spontaneous Fisston half-11ves vo neutron number for the indicated elements (arrows refer to experimental limits determined in this work, open circles refer to parabolic fit: see Section $v-c)$.

F1g. 4. Alpha-particle energies vs neutron number for the indicated elements.

FIg. 5. Electrospraying apparatus.

Fig. 6. Schematic of the Recoil collection Irradiation System.

Fig. 7. Schematic of the Rotating Drum Irradiation System.

Fig. 8. Relative elution positions of callfornium, berkelium, curium,

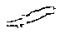
and americium compared with their lanthenide howologites on Dowex-50 resin and citrate eluant at $87^{\circ} \mathrm{C}$ (data from referenfe 66).". .

Fig. 9. Flow chart for Bk(TV) separation Using TTA.

Fig. 10. Flow chart for Bk(IV) separacion using HDEHP.

Fig. 1l. Flow chart for the separation of curfum and americtum (transplutonium elements) from an ain-inum caccher foll.

fig. 12. Samples of fission tracks in muscovite mica.

Fig. 13. Alpha"spectrum of the ${ }^{242} \mathrm{Ca-milking} \mathrm{sample} \mathrm{from} 11_{B}+235_{U}$ at $60 \mathrm{MeV}$ (30-day count).

Fig. 14. A typlcal gamma-ray spectrum of a berkelium sample from $11_{B}+235 \mathrm{U}$. Fig. 15. A typical Bana-ray spectrua of a $\mathrm{Co} / \mathrm{Am}$ sample from $11_{\mathrm{B}}+235_{\mathrm{U}}$. F1g. 16. Decay cur:e of curiun $k_{a_{1}}+k_{2} x$-rays froi the berkelium chemical fraction $\left(11_{\mathrm{B}}+{ }^{1235} \mathrm{U}\right)$. 


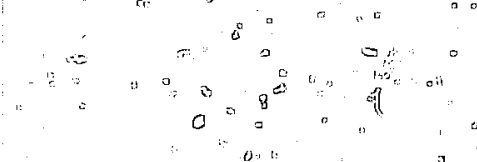

0
$\cdots "$ a $\rightleftharpoons=$
$=72-$

Fig: 17. Excitation functión for ${ }^{242} \mathrm{Bk}$ rom $11_{\mathrm{B}}+235_{\mathrm{U}}$ F1g. 18. Excitation function for ${ }^{242}$ gk frot $15 \mathrm{~N}+{ }^{232} \mathrm{Th}$. ec.

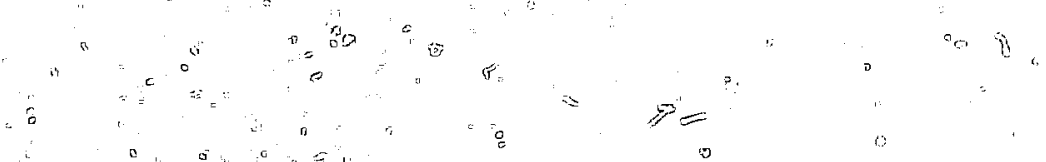

$\because$

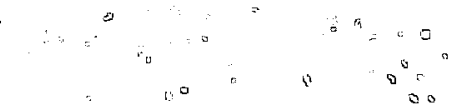

30000000

to $\quad 4 i^{\circ}=$

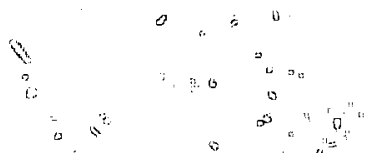

$4 \quad+1=0$

6

N

$y$

0

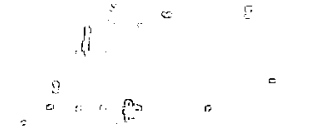

":

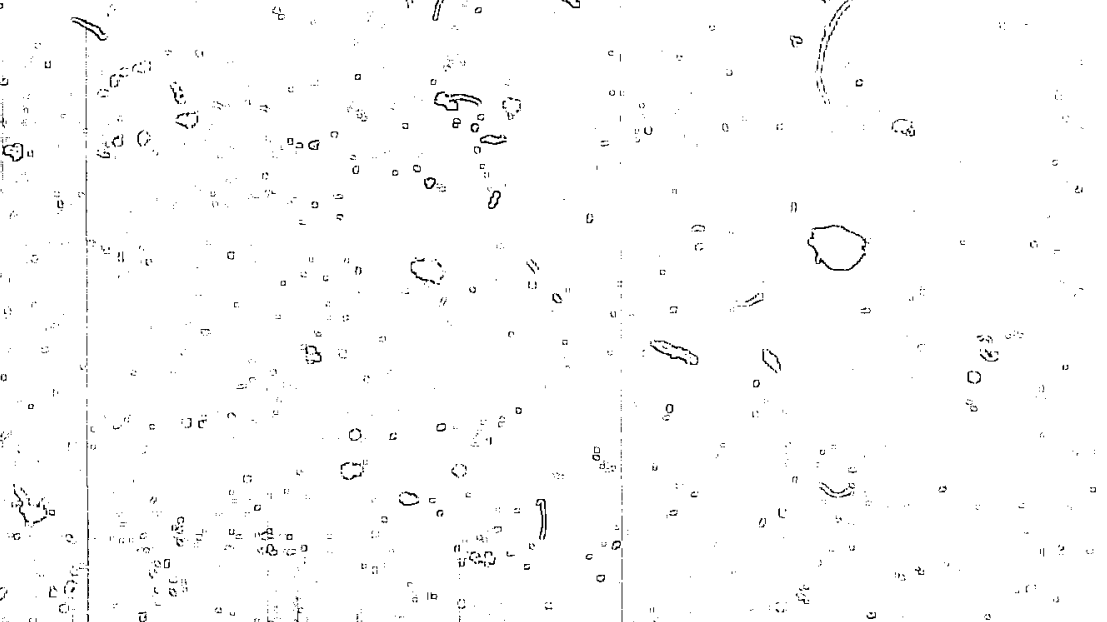




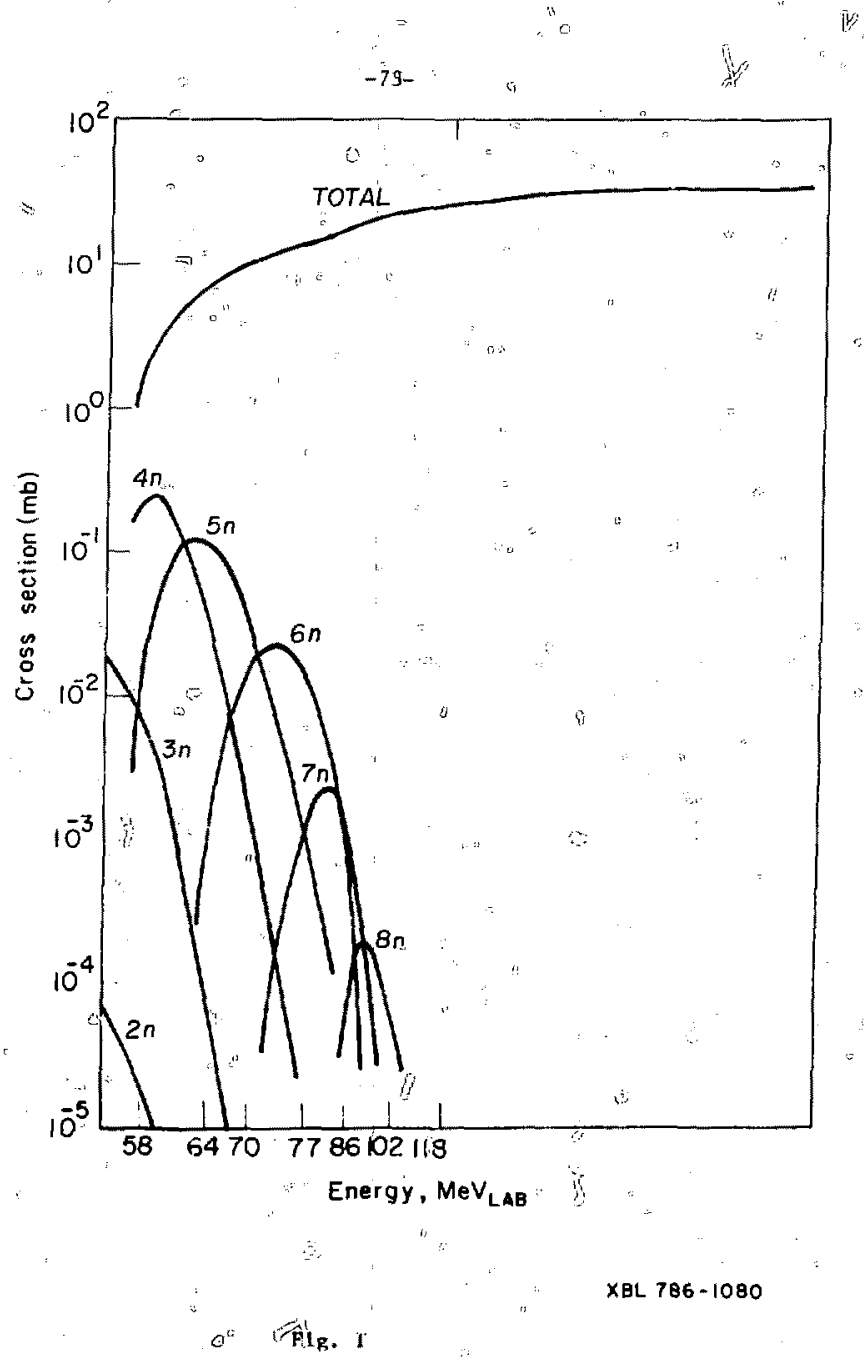

ie

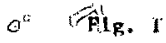

(3) 


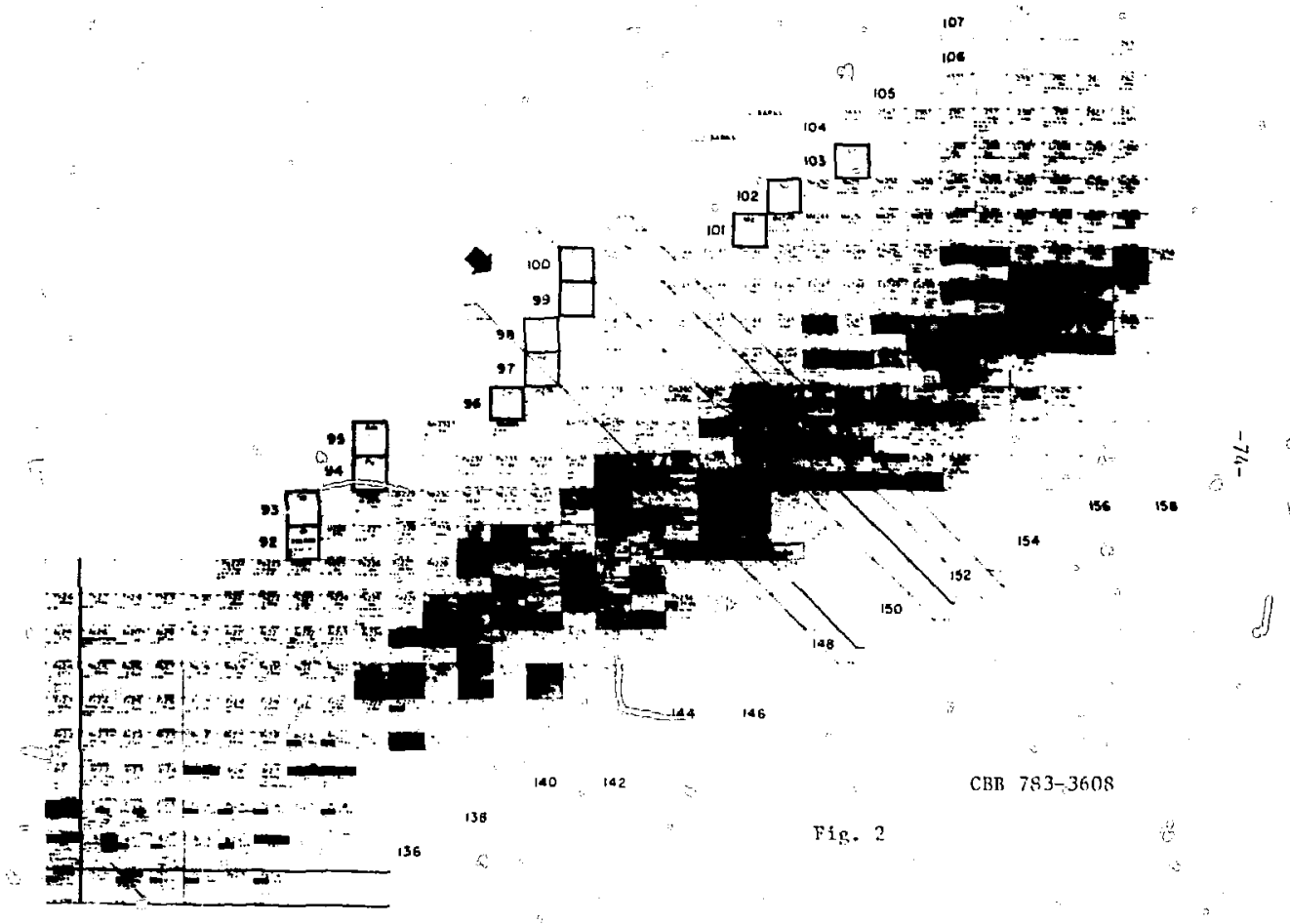




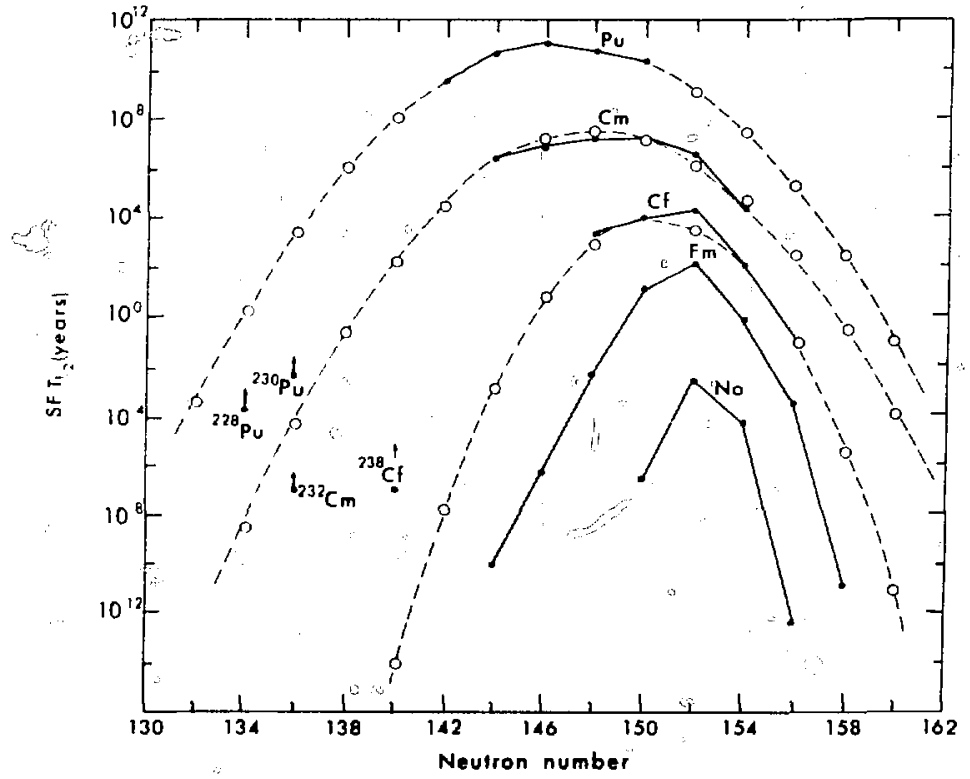

XBLY66-B233

Fin. ? 


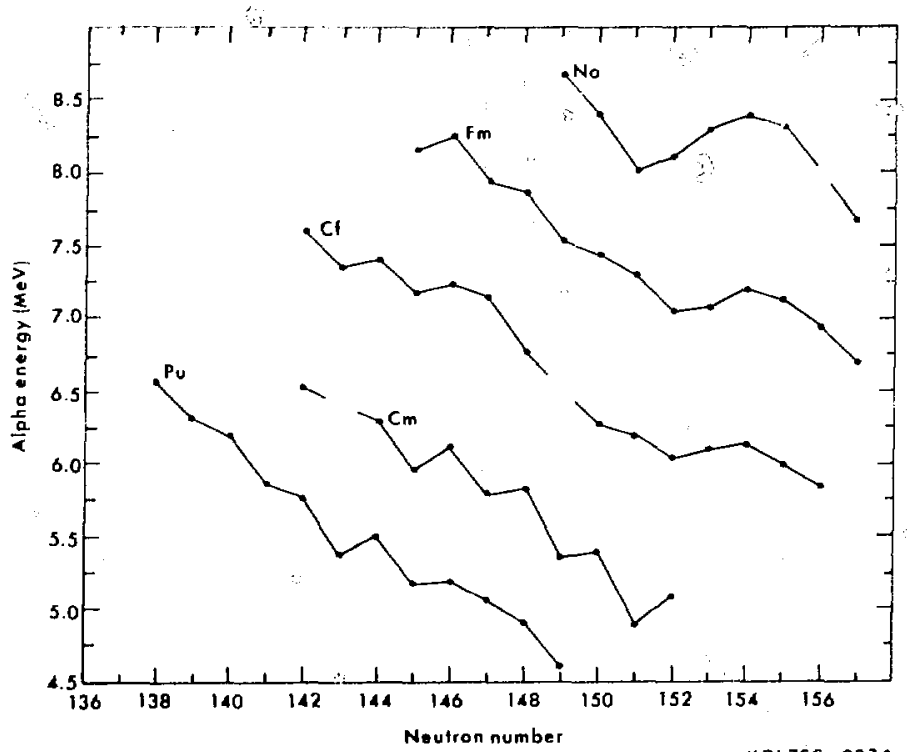

FIf. 4 


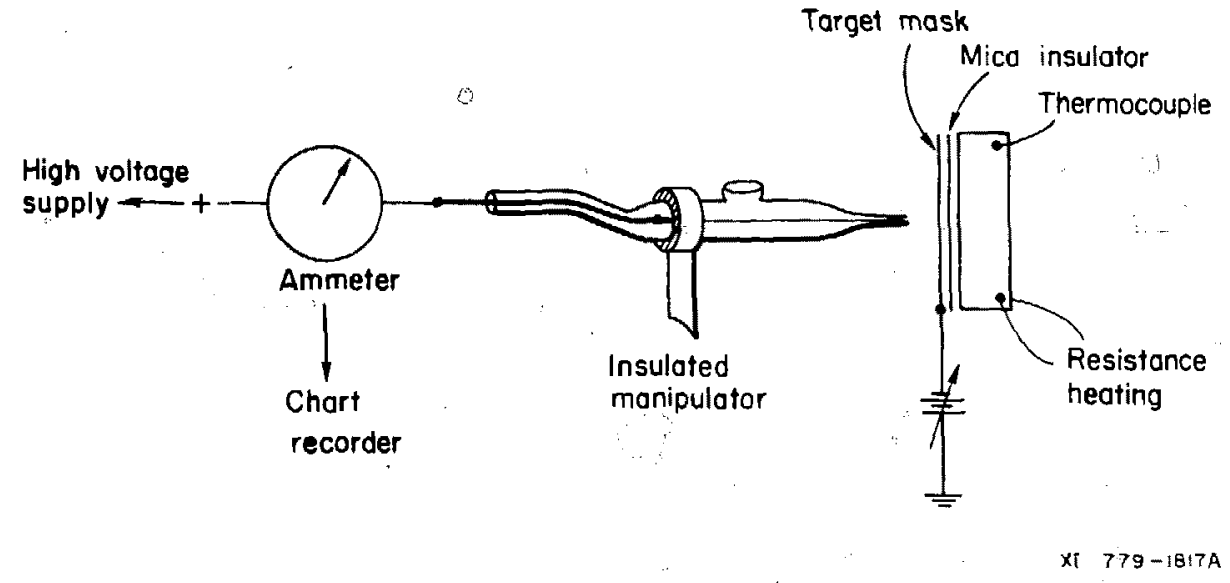

FIg * 5 


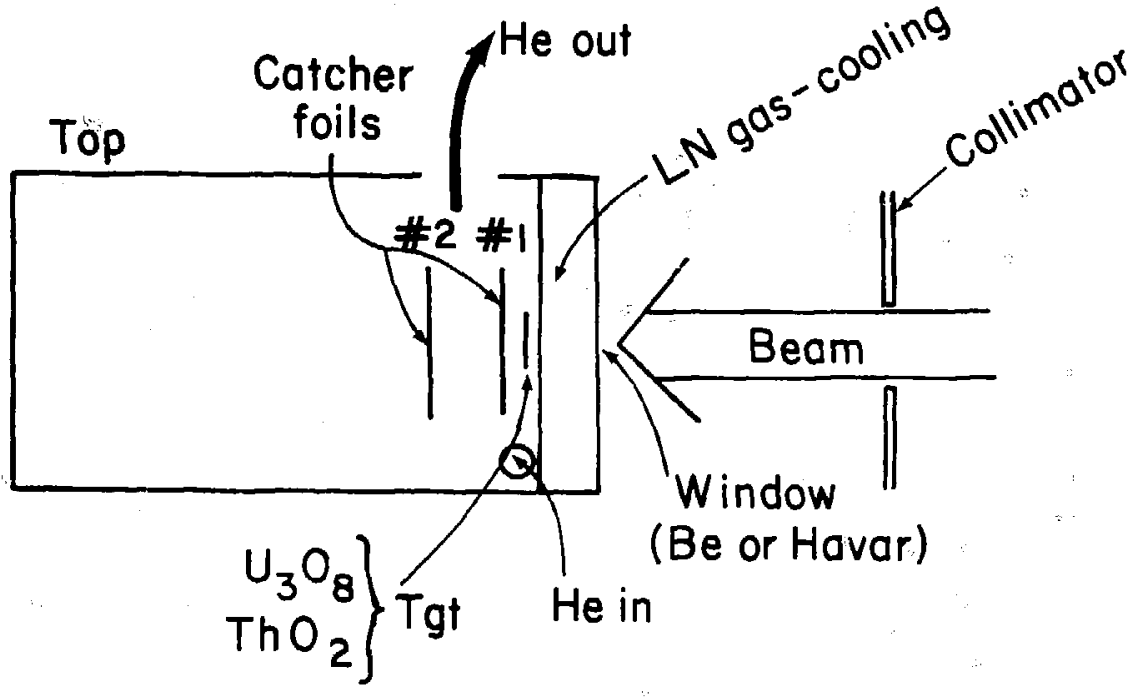


9

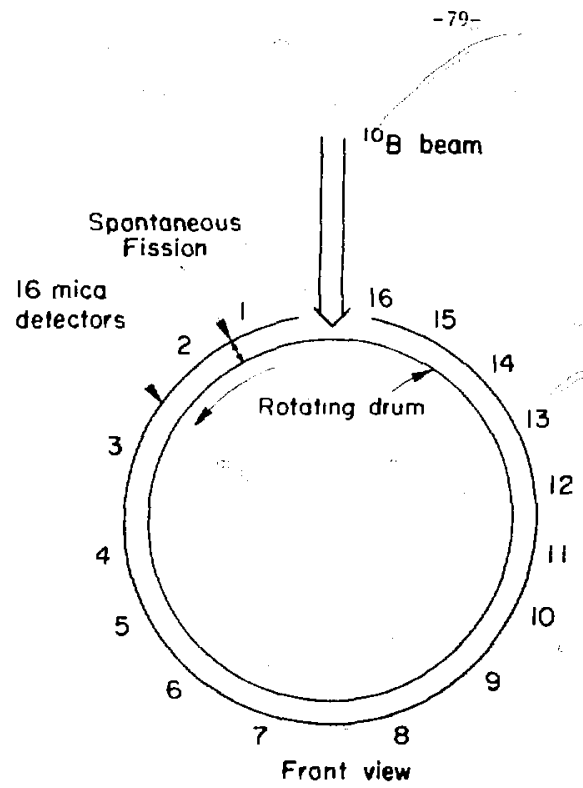

Fission tracks -
2

Mics

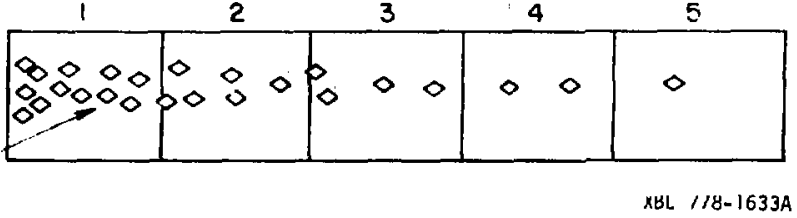

Fig. 7 


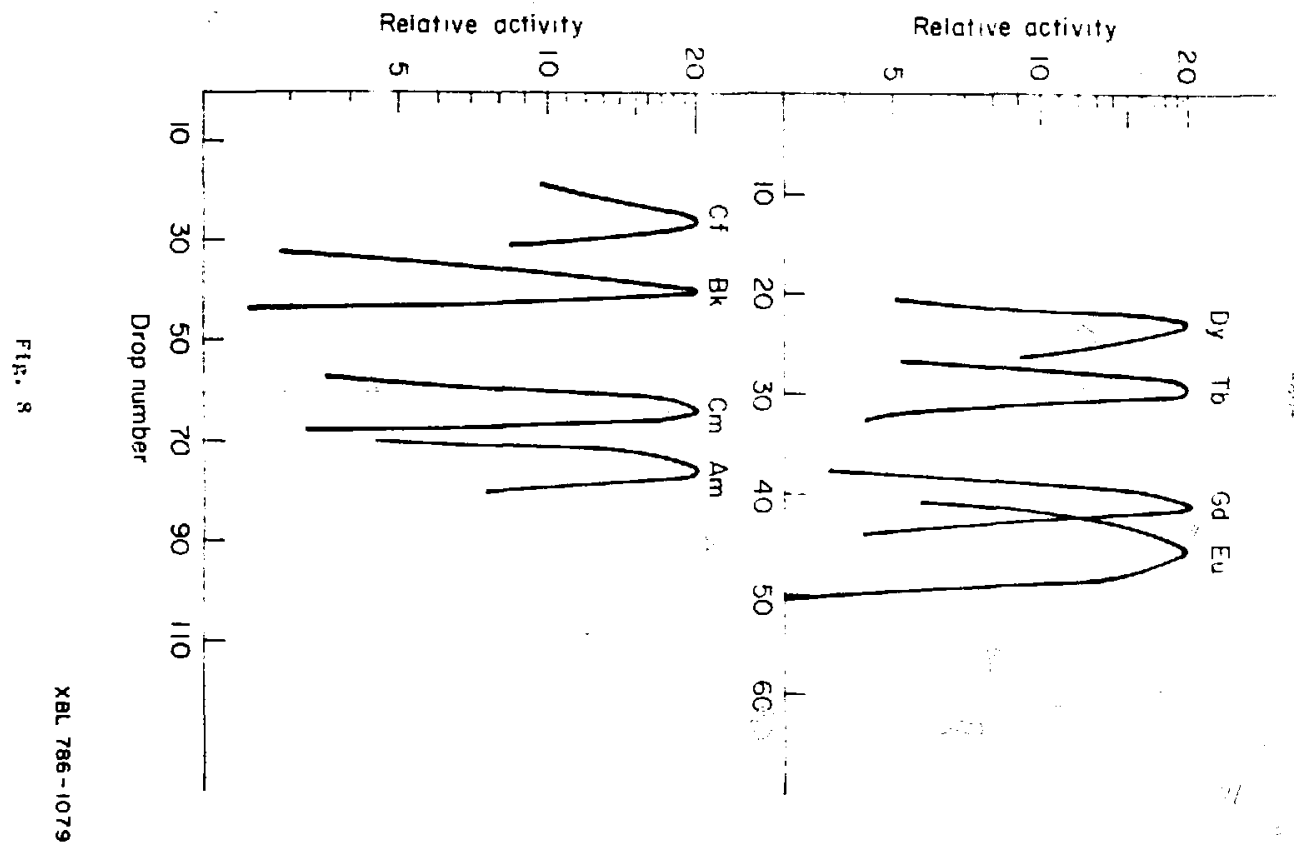




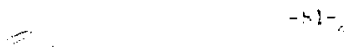

\section{TTA SOLVENT EXTRACTION OF BK}

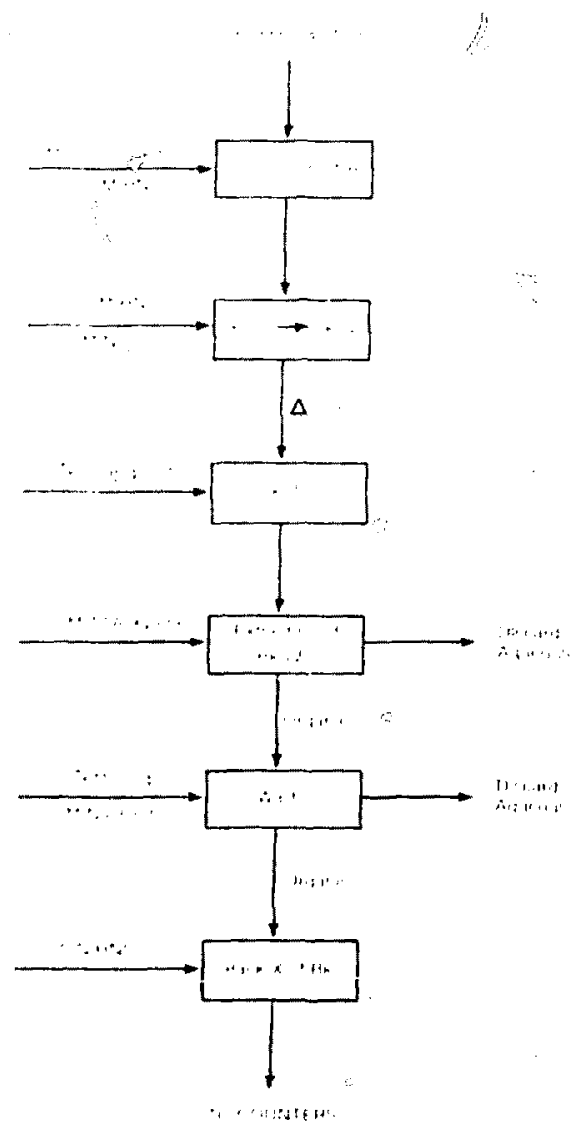




\section{HDEHP SOLVENT EXTRACTION OF BK}

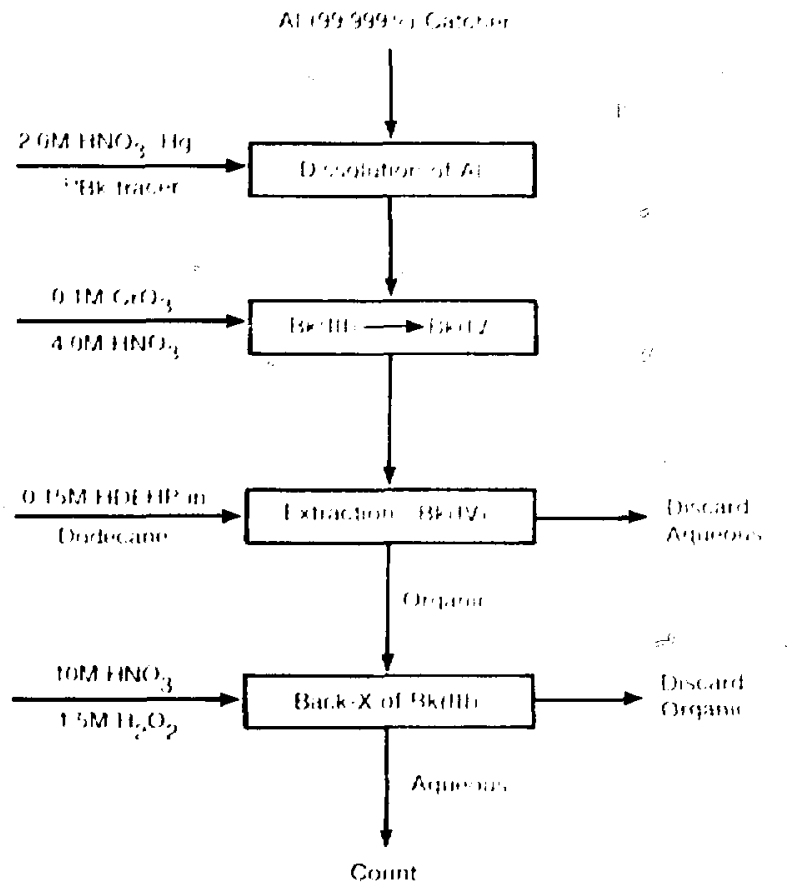

XBL $786-1075$

F1s. 10 
HWAR'

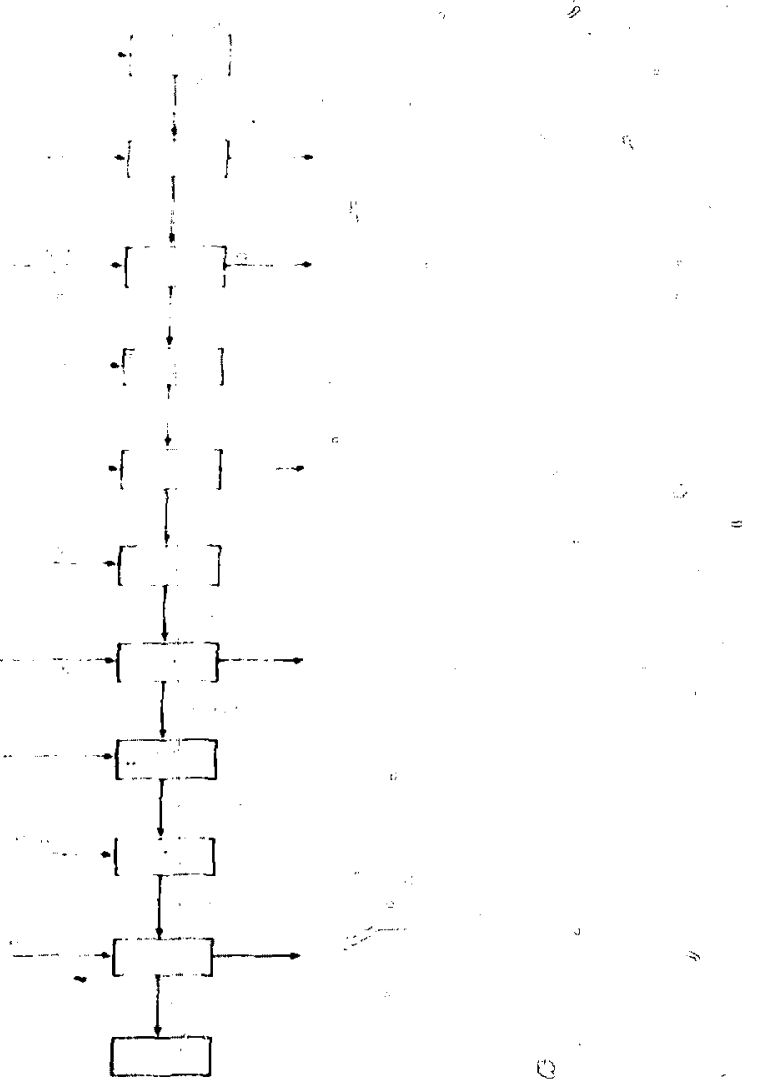

XBL $706-107 ?$

FIg. 11 
$\sqrt{6} / 4-$

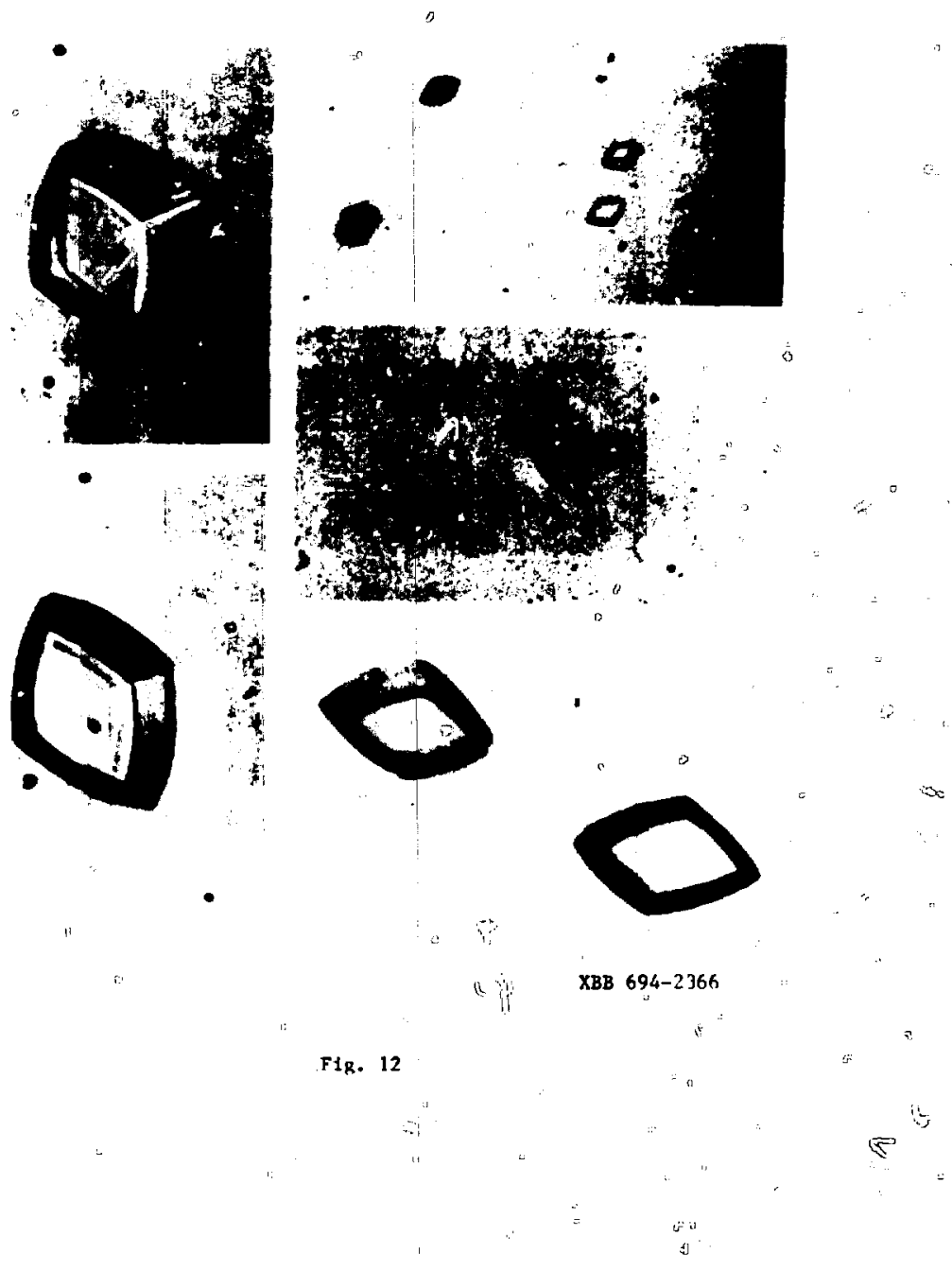


CLUNTS PER. CHASNEL.

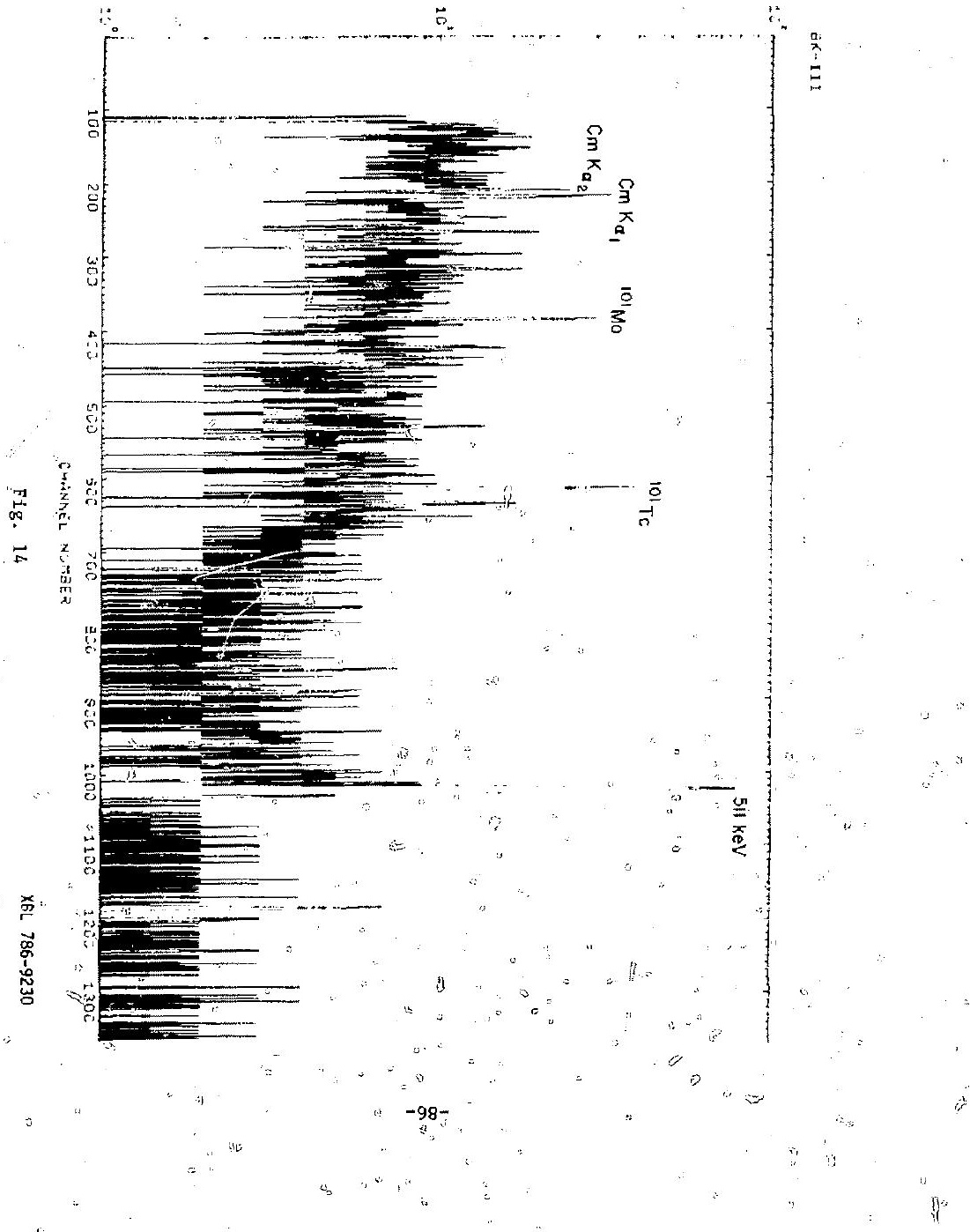



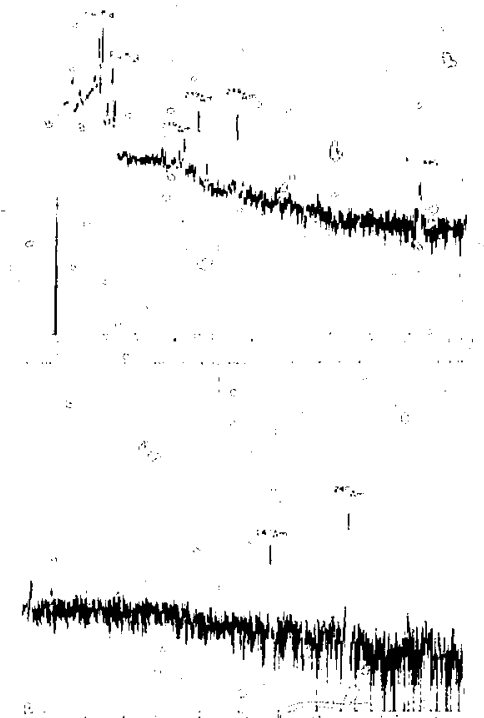

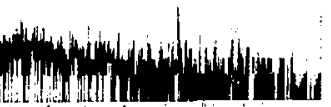

Fig. 15 


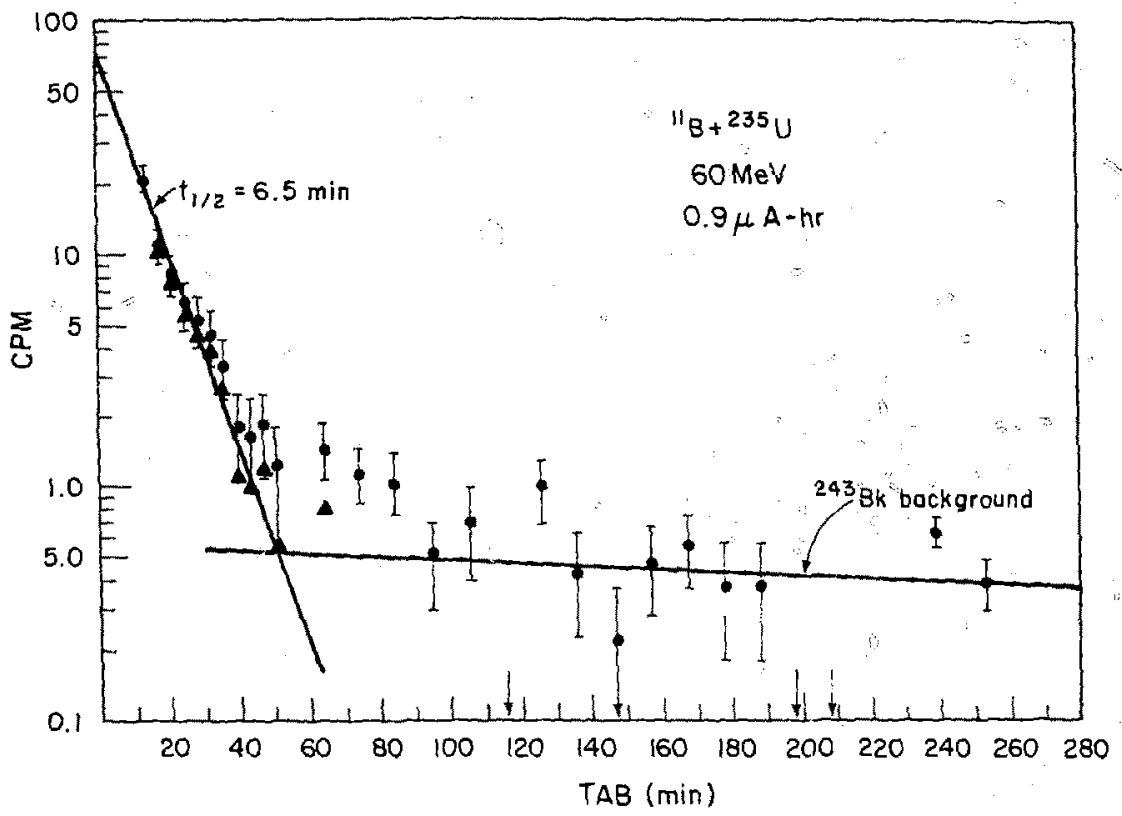




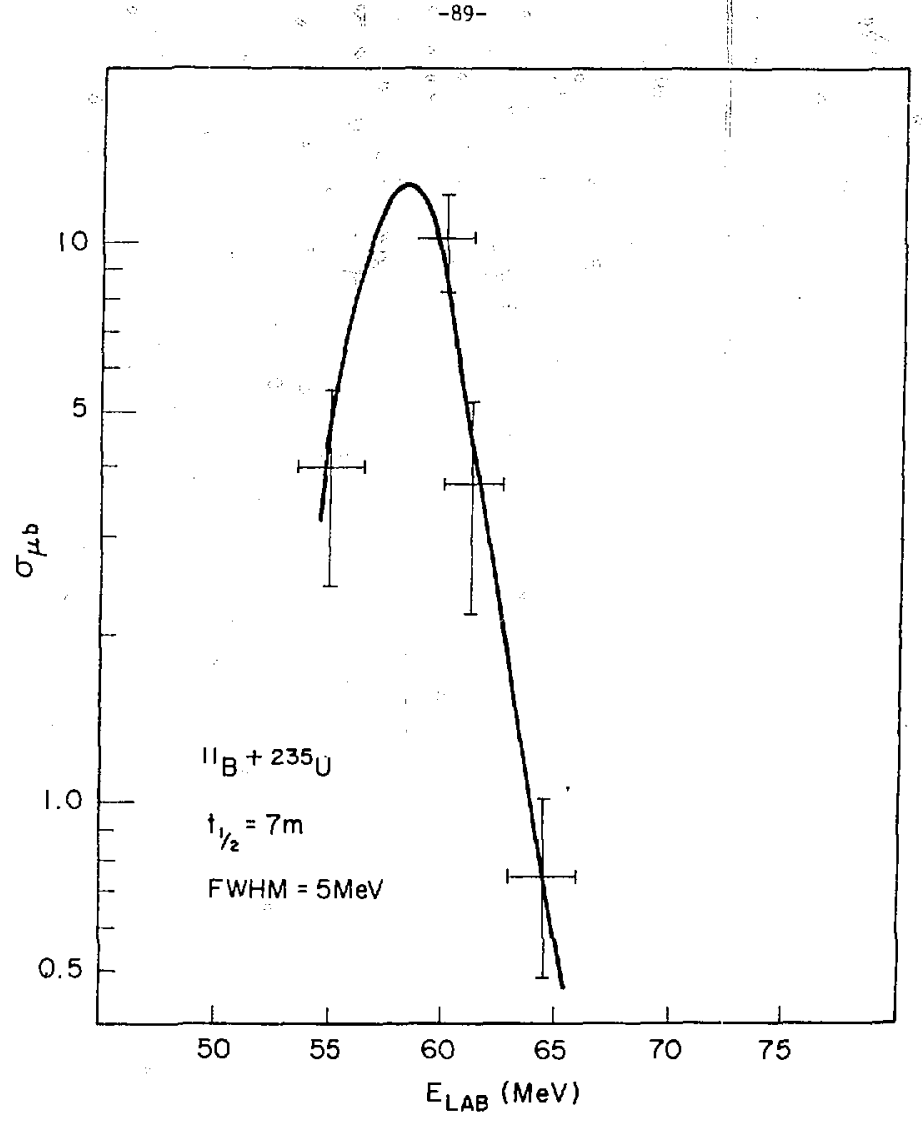

XB! $786-9223$

Fig. 17 


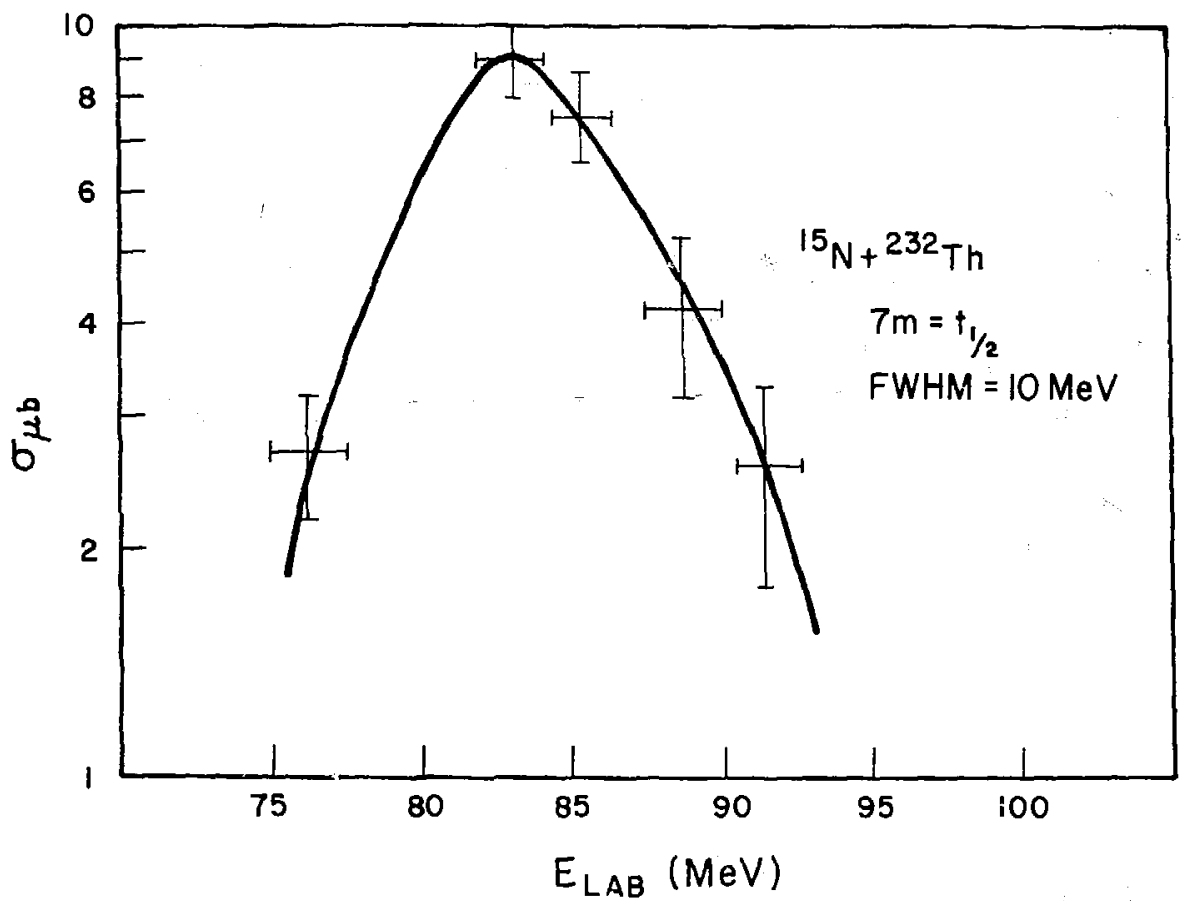

